\title{
Zigzag Molecules from Pyrene-Modified Carbazole Oligomers: Synthesis, Characterization and Application in OLEDs
}

\author{
Zujin Zhao, ${ }^{a}$ Xinjun Xu, ${ }^{\text {b }}$ Hongbo Wang, ${ }^{\text {a Ping Lu, }}{ }^{\text {,a }}$ Gui Yu ${ }^{\text {b }}$ and Yunqi Liu, ${ }^{*, b}$ \\ ${ }^{a}$ Department of Chemistry, Zhejiang University, Hangzhou 310027, People's Republic of China \\ ${ }^{b}$ Laboratory of Organic Solids, Institute of Chemistry, Chinese Academy of Sciences, Beijing 100080, \\ People's Republic of China
}

\section{Supporting Information}

CONTENTS:

General information.

Fabrication and characterization of OLEDs

Figure S1-S48. NMR and mass spectra.

Figure S49-S54. $J$ - $V$ - $L$ characteristics and efficiency spectra of OLEDs
Page

S2

S2

S3-S26

S27-S28

* Corresponding author. Tel.: + 86-571-879- 52543.

E-mail address: pinglu@zju.edu.cn (P. Lu). 
General information. NMR spectra were recorded using a $500 \mathrm{MHz}$ FT NMR spectrometer. Five percent w/v solutions in chloroform- $d$ were used to obtain NMR spectra. TMS was used as an internal standard. Mass spectroscopic (MS) measurements were carried using matrix-assisted laser desorption ionisationtime-of-flight (MALDI-TOF) technique. UV-visible absorption and fluorescence emission spectra were performed in dilute THF or cyclohexane solutions $\left(\sim 1 \times 10^{-6}\right)$ in $1 \times 1 \mathrm{~cm}$ quartz cells or in thin neat films spin-coated on quartz substrates at room temperature. Thermal stabilities were determined by thermal gravimetric analyzer with heating rate of $10{ }^{\circ} \mathrm{C} / \mathrm{min}$ under $\mathrm{N}_{2}$. Electrochemical measurements were performed using Pt working electrode, an auxiliary Pt electrode, and an Ag/AgCl reference electrode at room temperature. The solvents were distilled before used. Commercially available reagents were used without further purification unless otherwise.

Fabrication and characterization of OLEDs. All the devices were fabricated on bare ITO substrates that were cleaned by detergent, deionized water, acetone, and ethanol. Poly(3,4-ethylenedioxythiophene):poly(4-styrenesulfonate) (PEDOT:PSS) was spin-coated on ITO glass, and was baked under $100{ }^{\circ} \mathrm{C}$ for 2 hours. All the oligomers were spin-coated onto PEDOT:PSS layer from their toluene solutions. Films of TPBI, $\mathrm{Alq}_{3}$ and $\mathrm{Al}$ cathodes were formed by vacuum deposition under a pressure of $3 \times 10^{-4} \mathrm{~Pa}$. The thickness of the thin films was monitored by a quartz crystal oscillator placed near the substrates and was calibrated ex situ by a surface profilometer. 
NMR and mass spectra of new compounds:
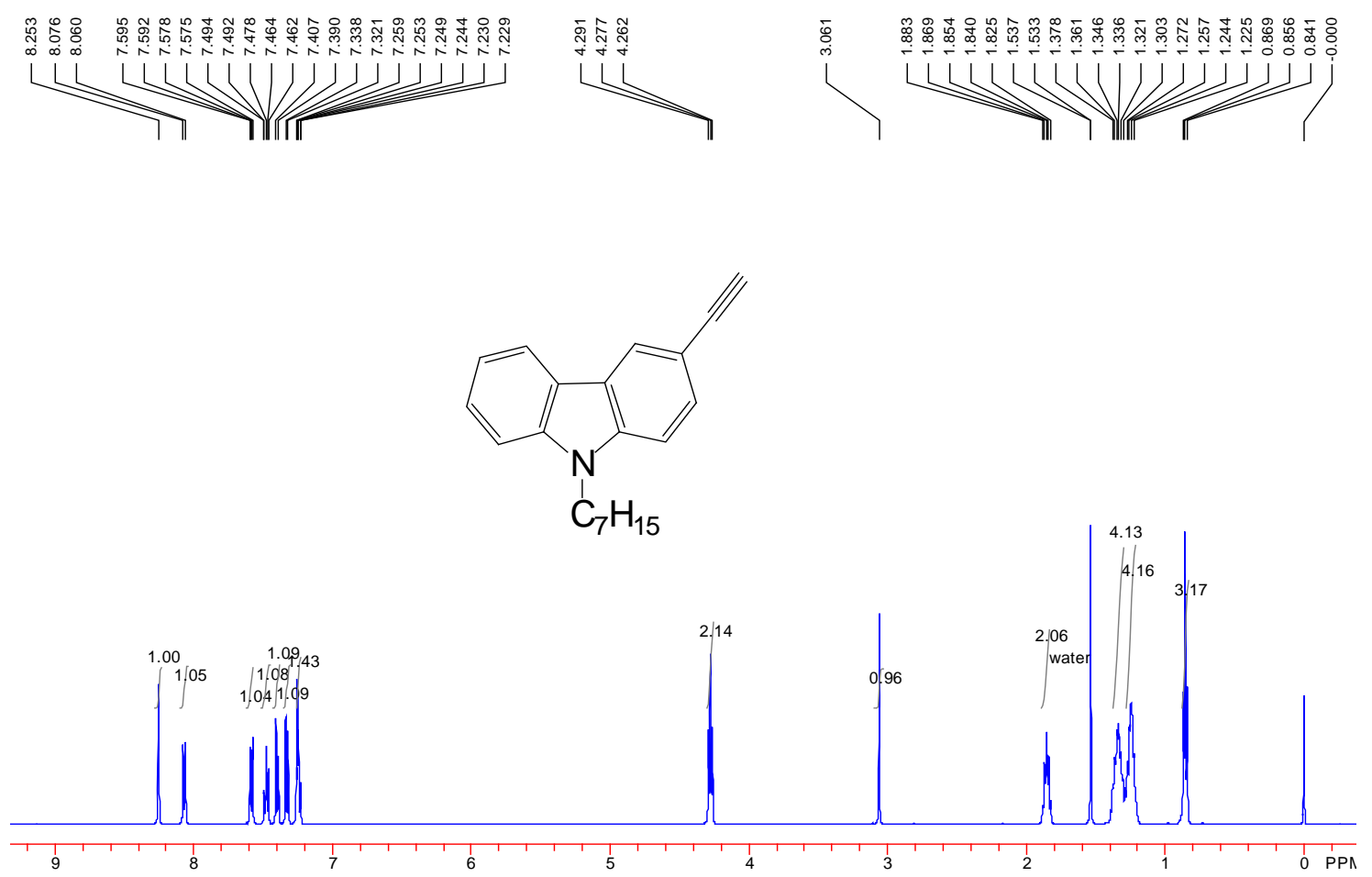

Figure S1: ${ }^{1} \mathrm{H}$ NMR spectrum of compound 3.
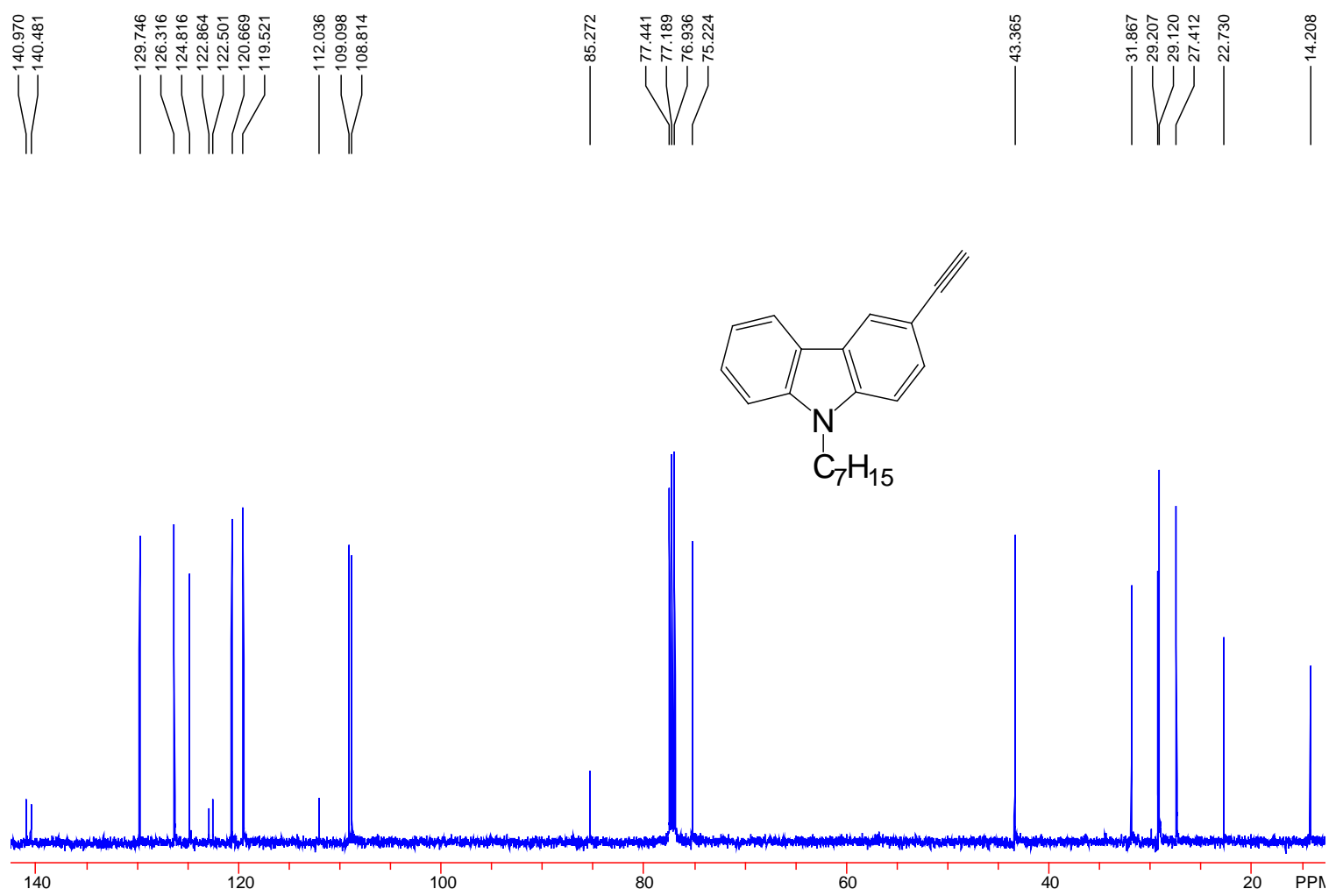

Figure S2: ${ }^{13} \mathrm{C}$ NMR spectrum of compound 3 . 


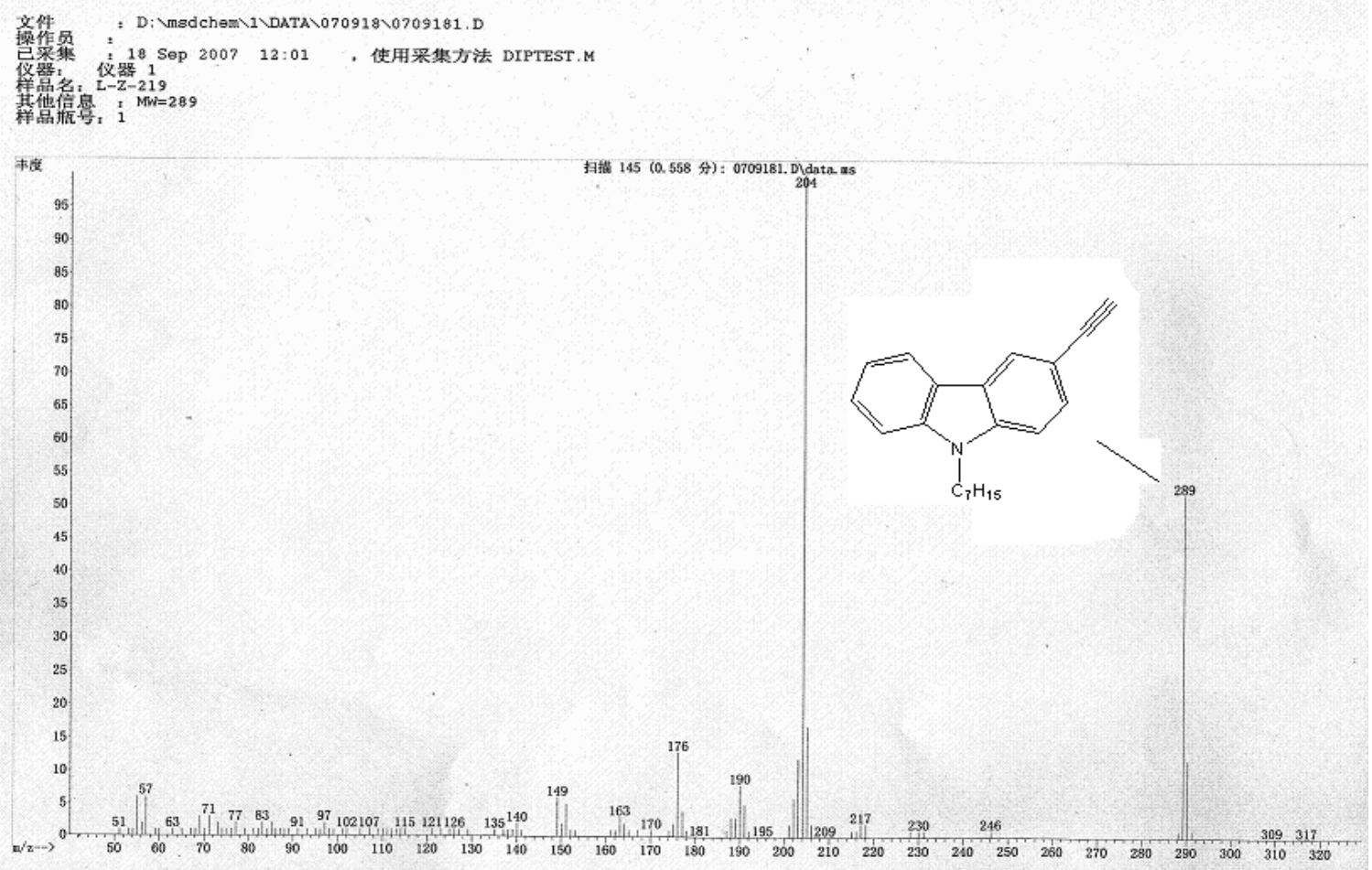

Figure S3: mass spectrum of compound 3.
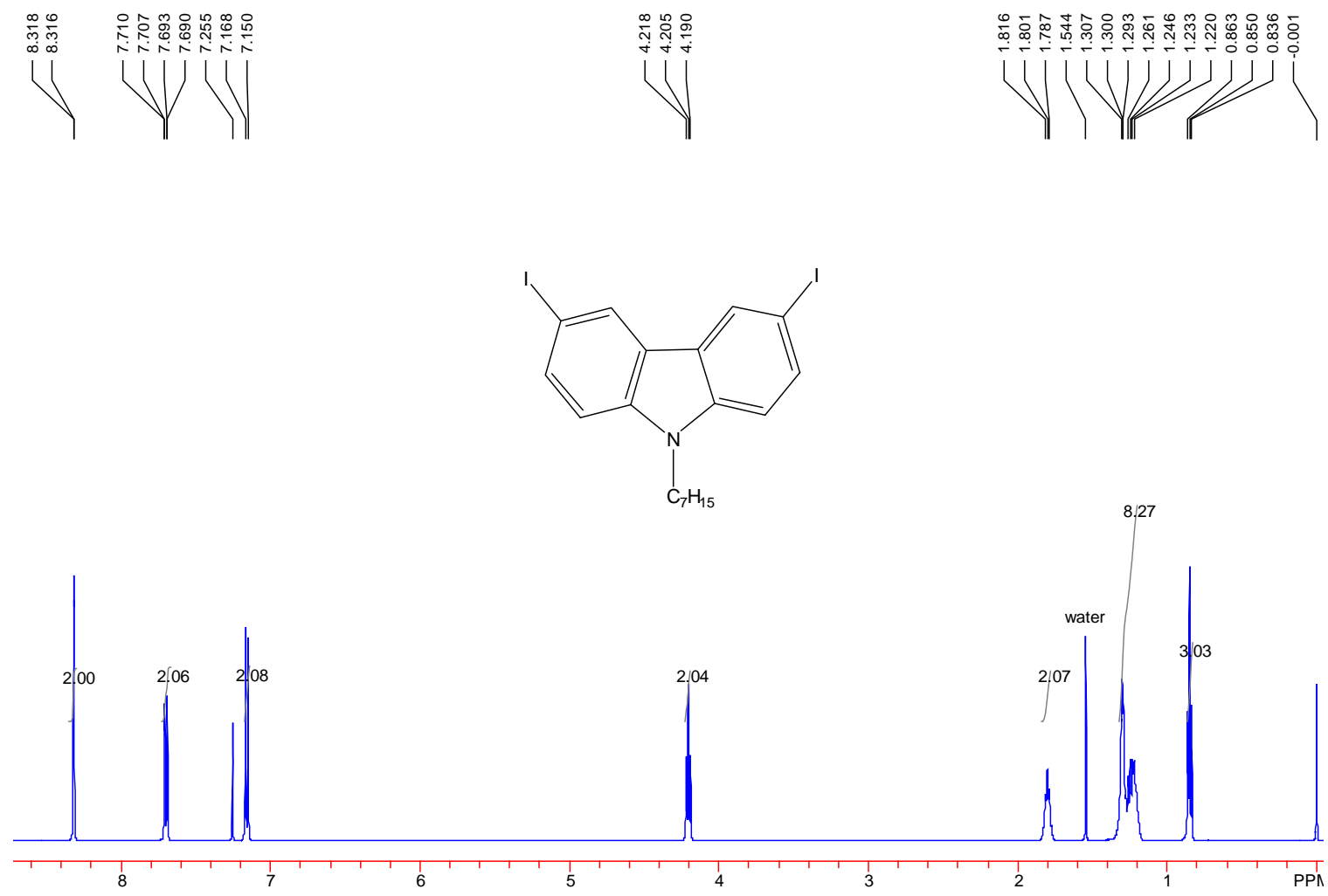

Figure S4: ${ }^{1} \mathrm{H}$ NMR spectrum of compound 4. 


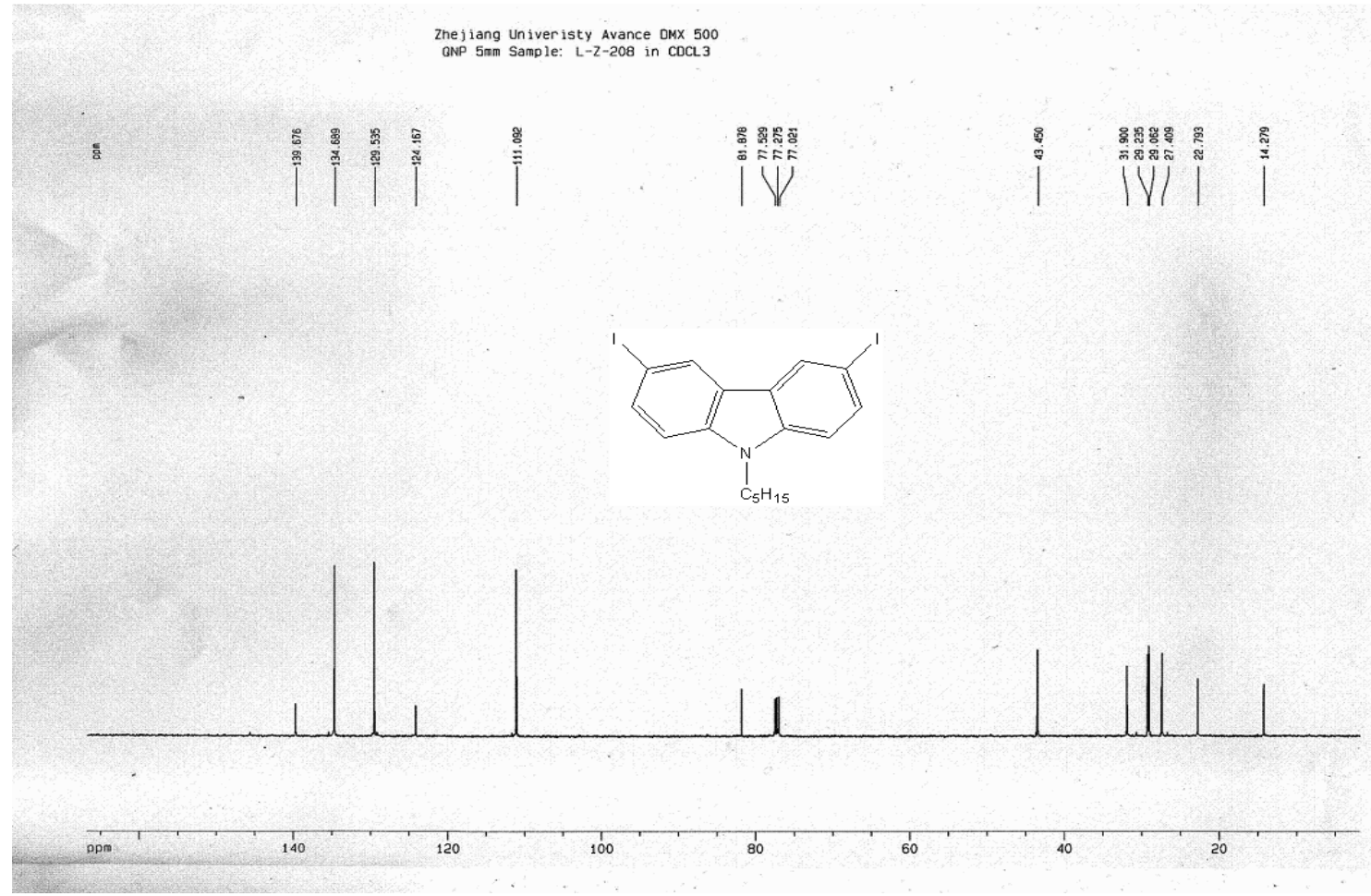

Figure S5: ${ }^{13} \mathrm{C}$ NMR spectrum of compound 4 .

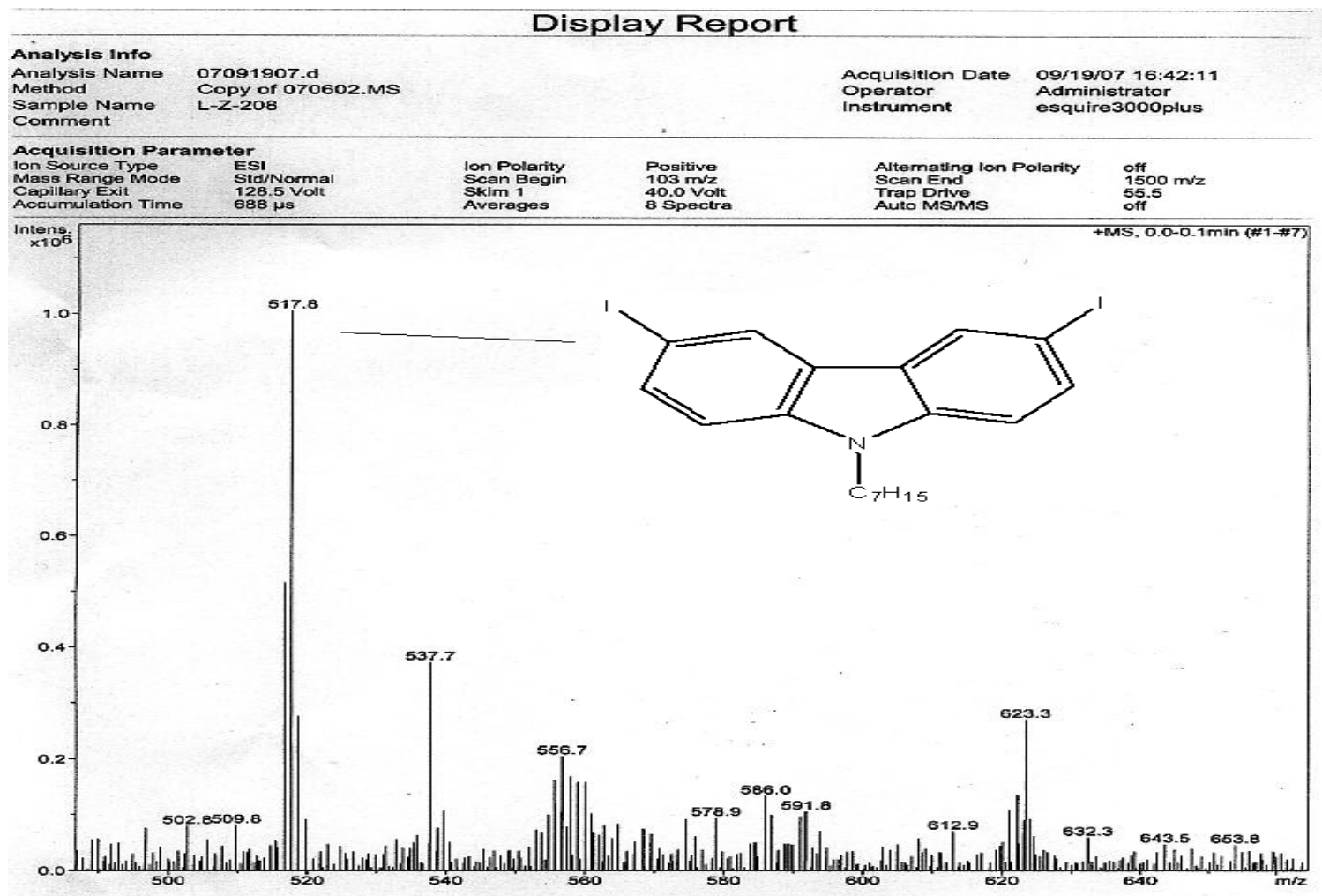

Figure S6: mass spectrum of compound 4. 

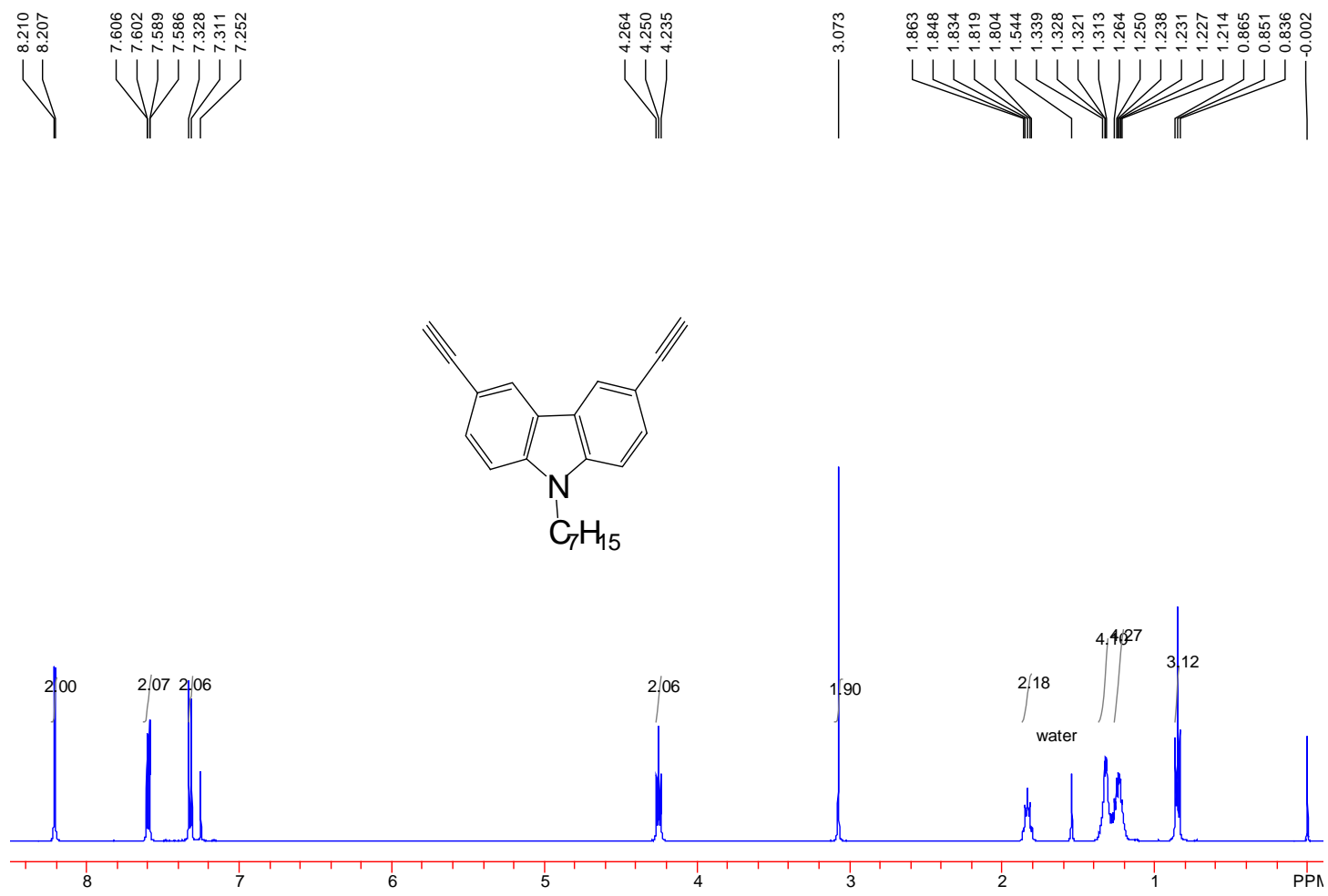

Figure S7: ${ }^{1} \mathrm{H}$ NMR spectrum of compound 6.
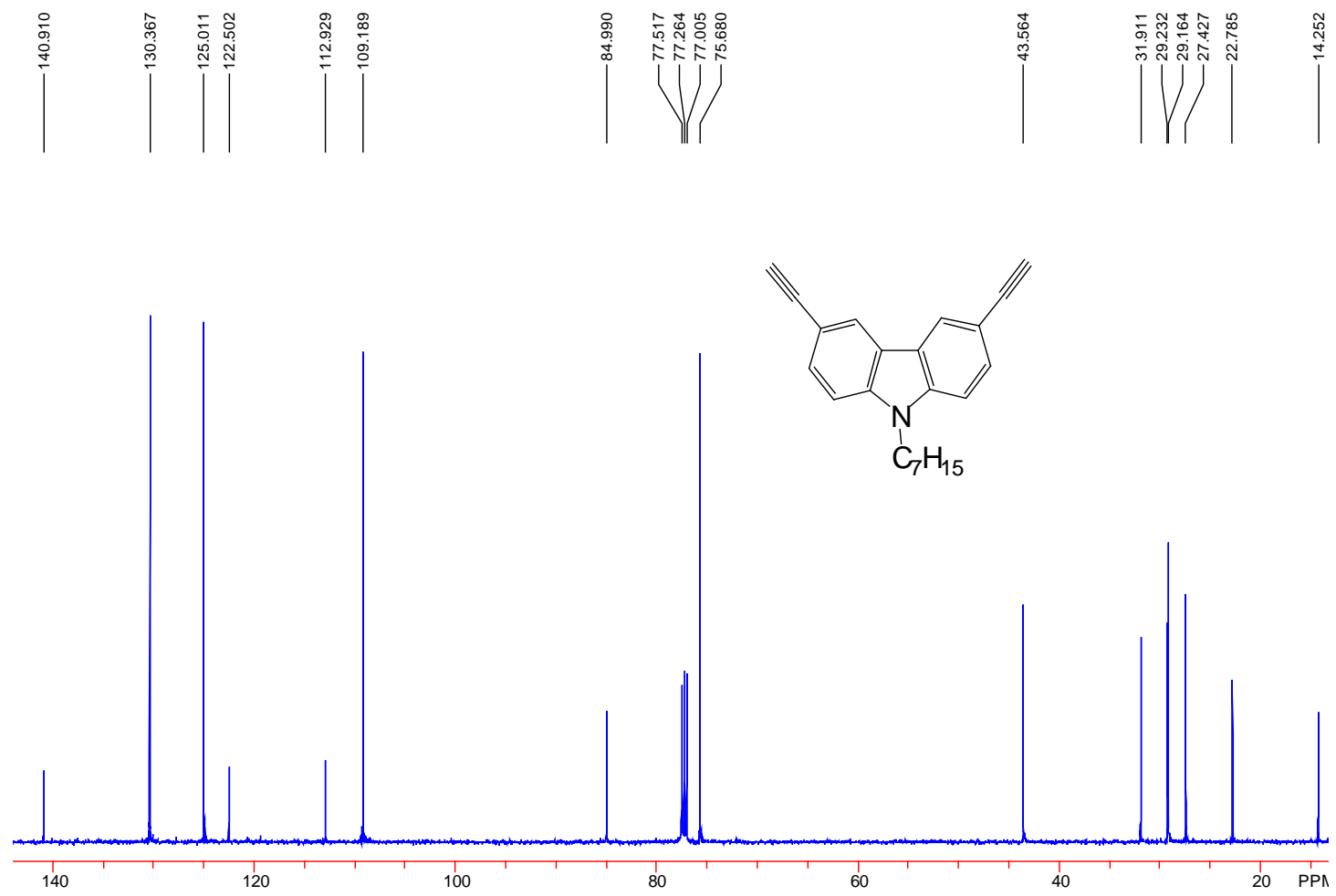

Figure S8: ${ }^{13} \mathrm{C}$ NMR spectrum of compound 6 . 


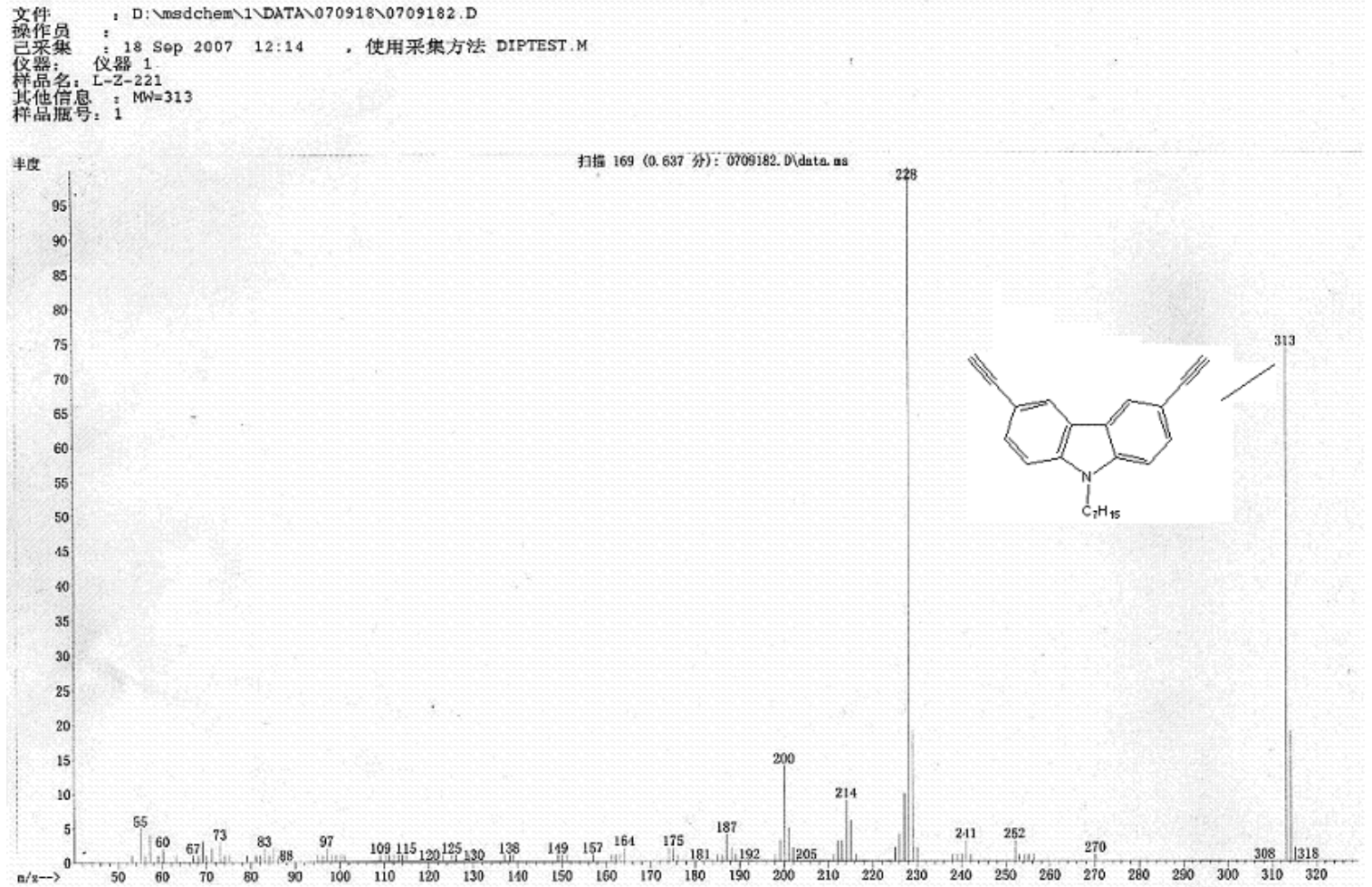

Figure S9: mass spectrum of compound 6.
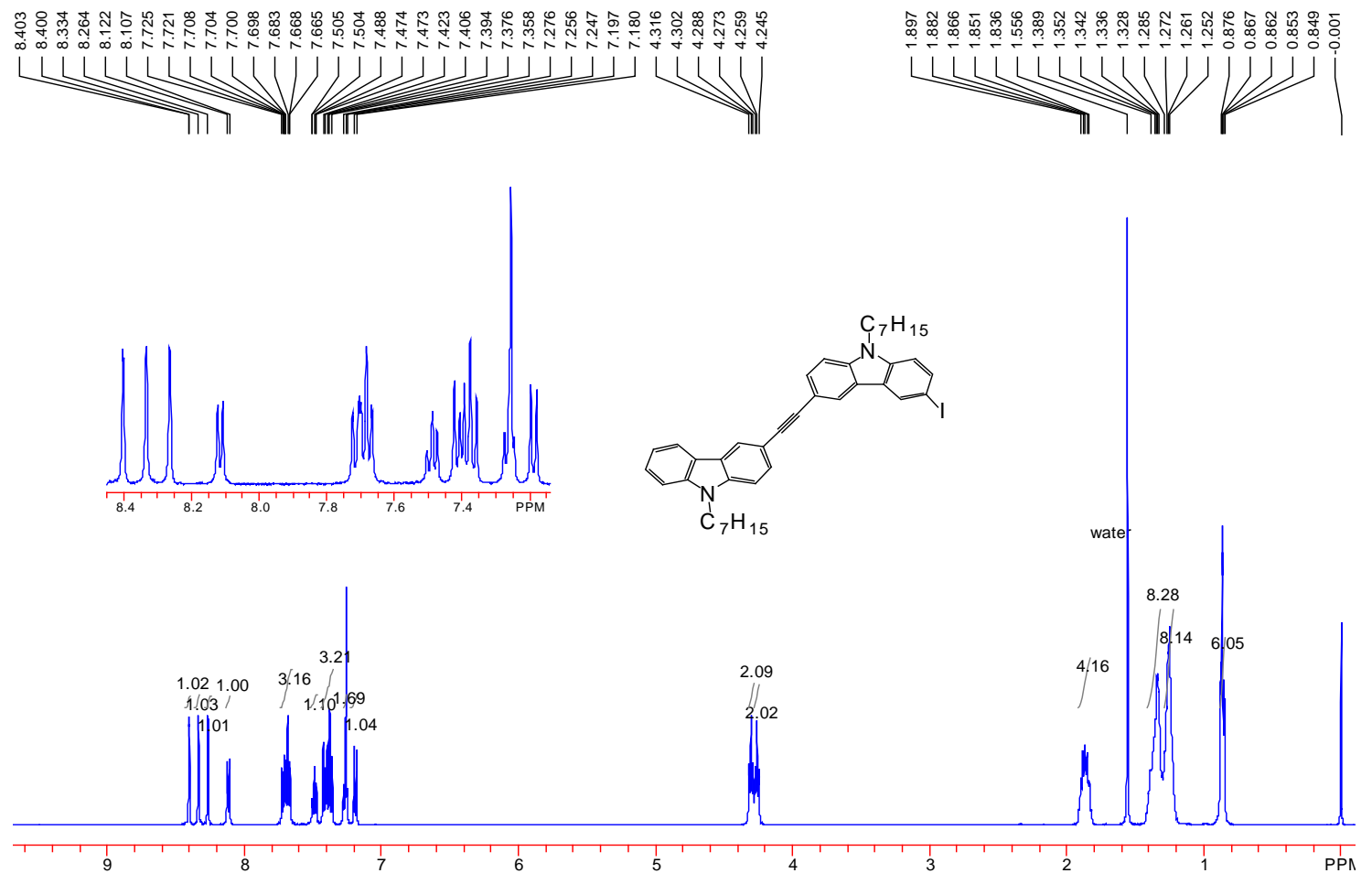

Figure S10: ${ }^{1} \mathrm{H}$ NMR spectrum of compound 7. 

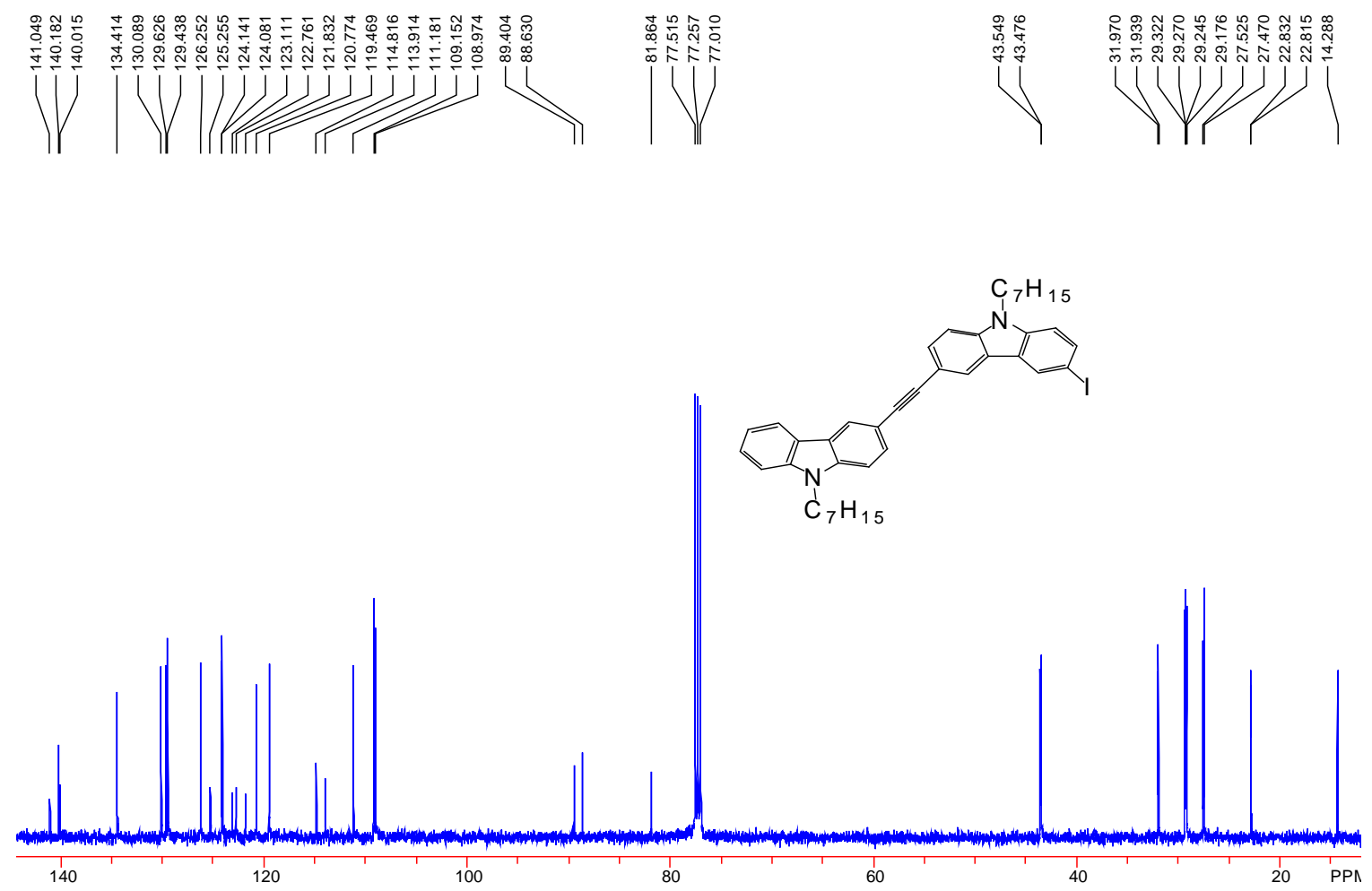

Figure S11: ${ }^{13} \mathrm{C}$ NMR spectrum of compound 7.

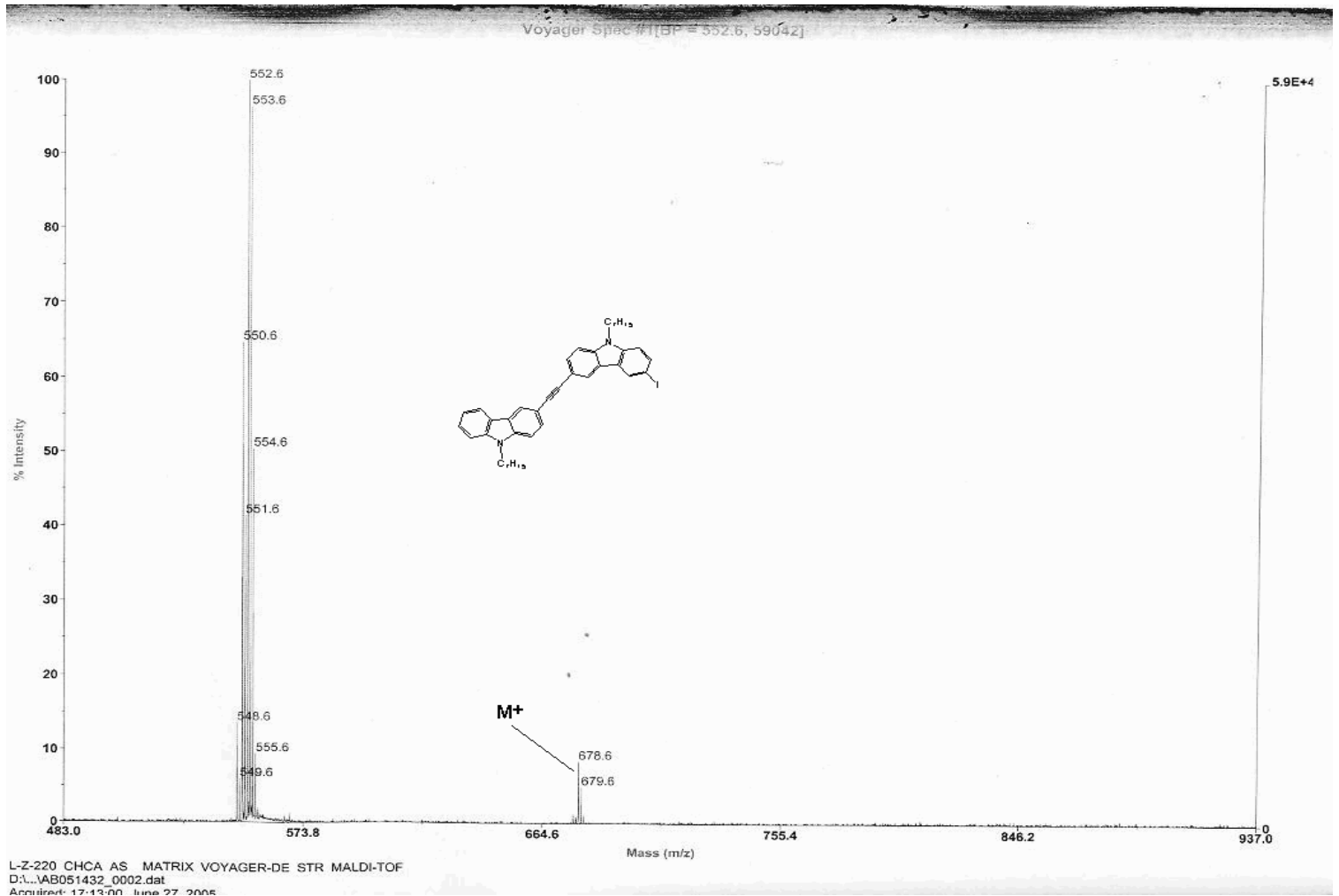

Figure S12: mass spectrum of compound 7. 

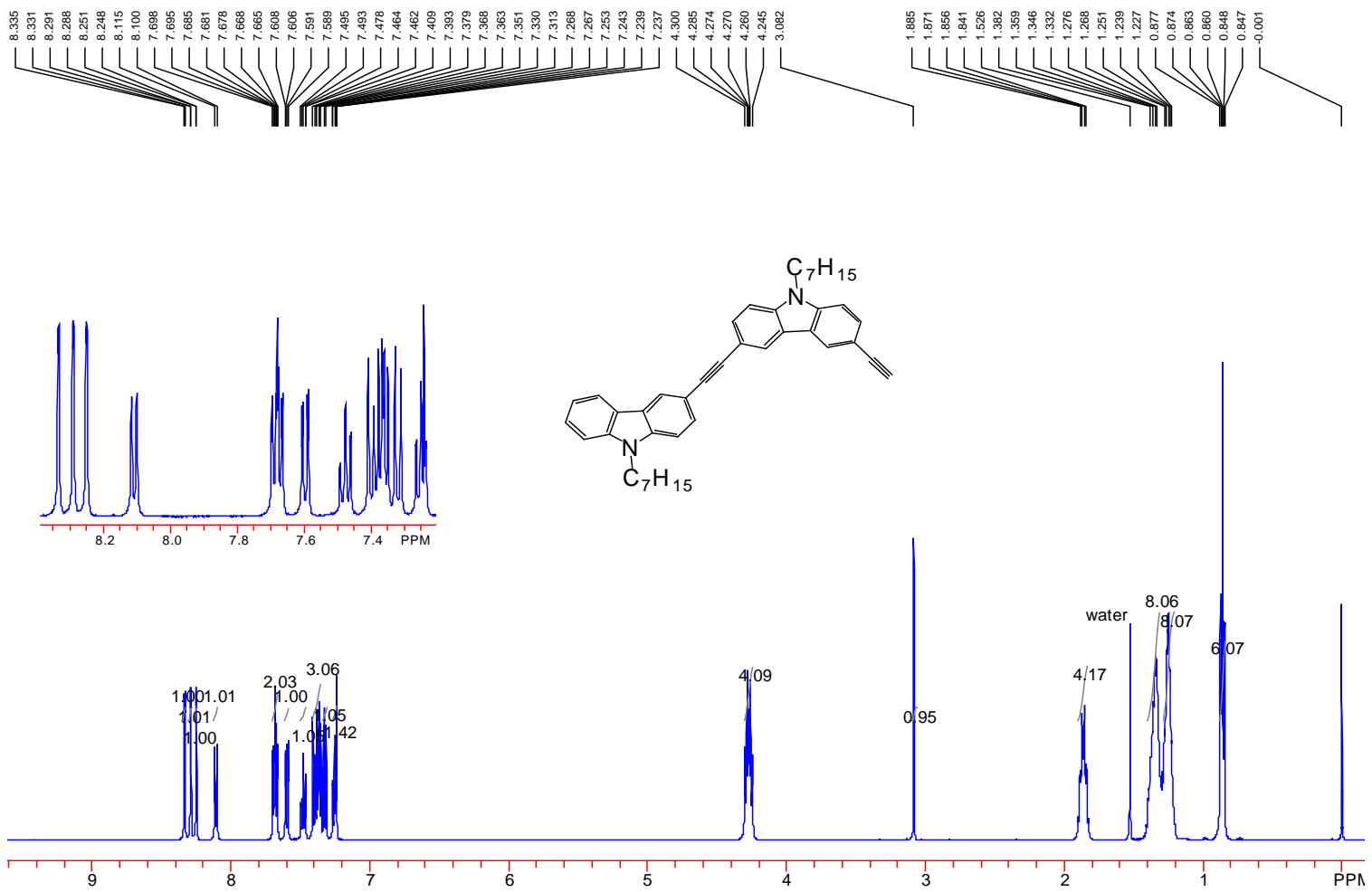

Figure S13: mass spectrum of compound 9.
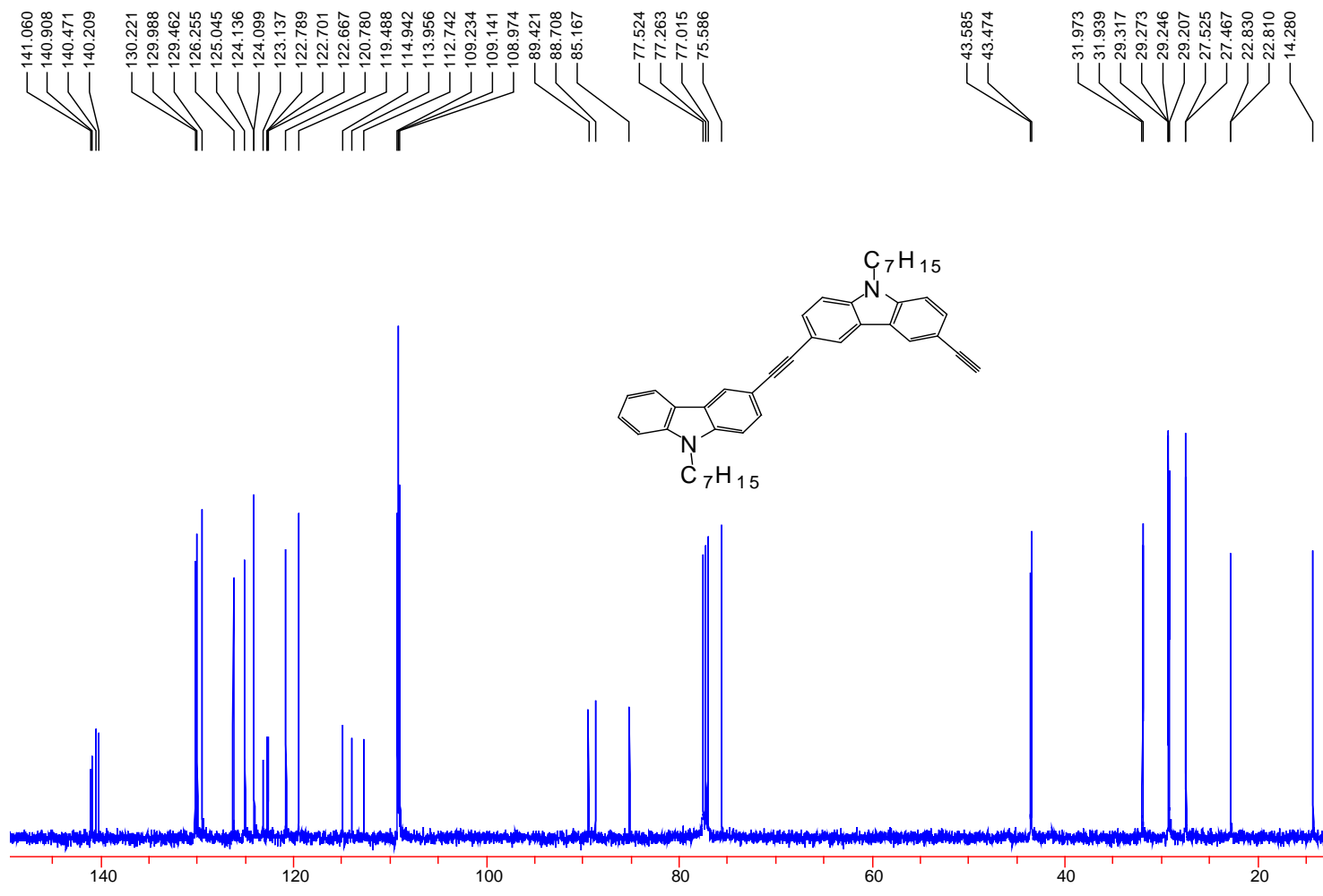

Figure S14: ${ }^{13} \mathrm{C}$ NMR spectrum of compound 9 . 


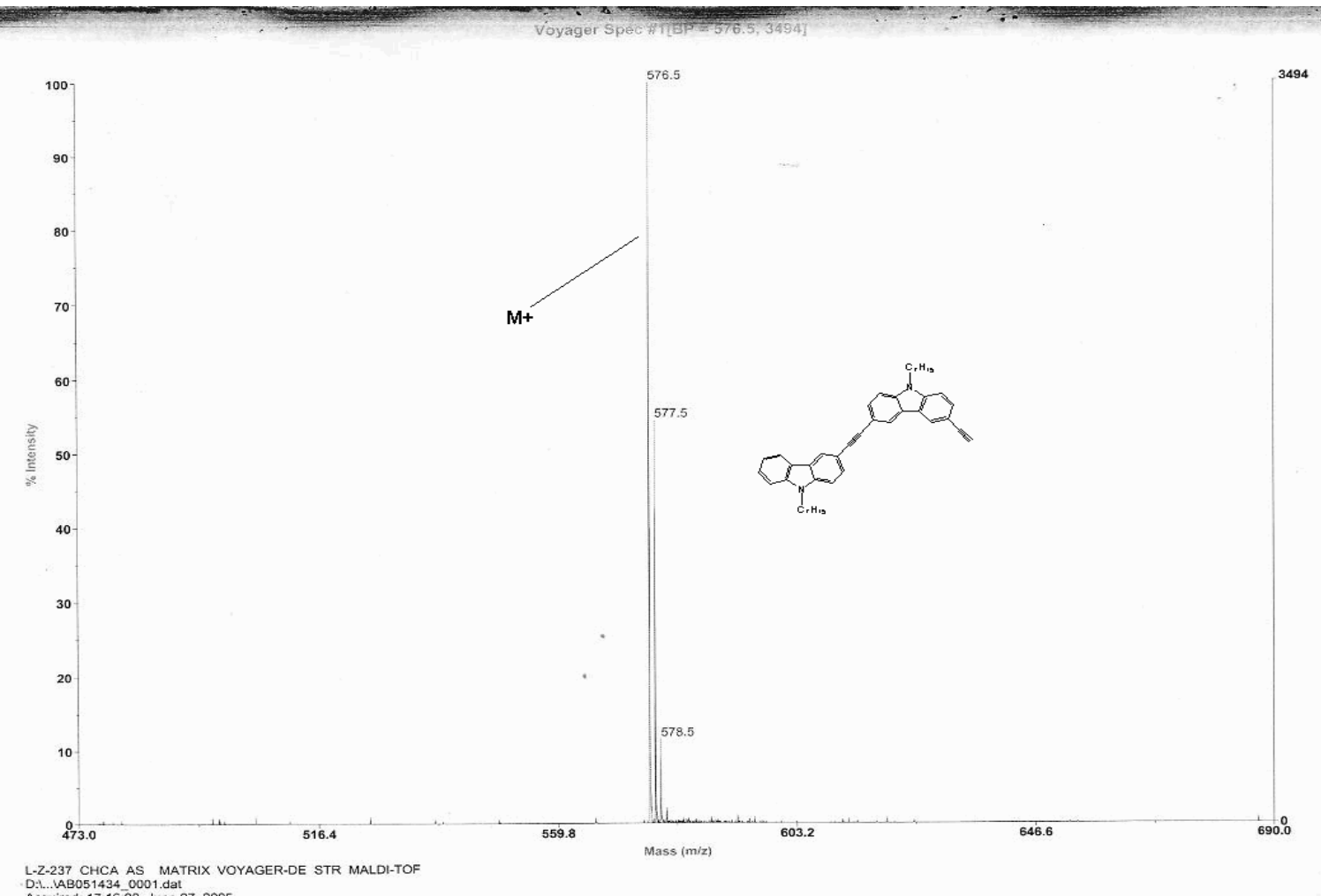

Figure S15: mass spectrum of compound 9.

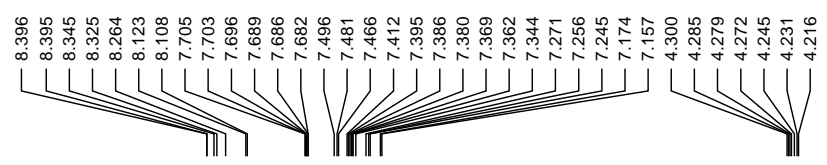

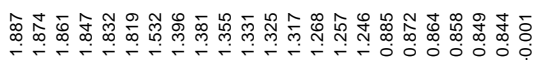
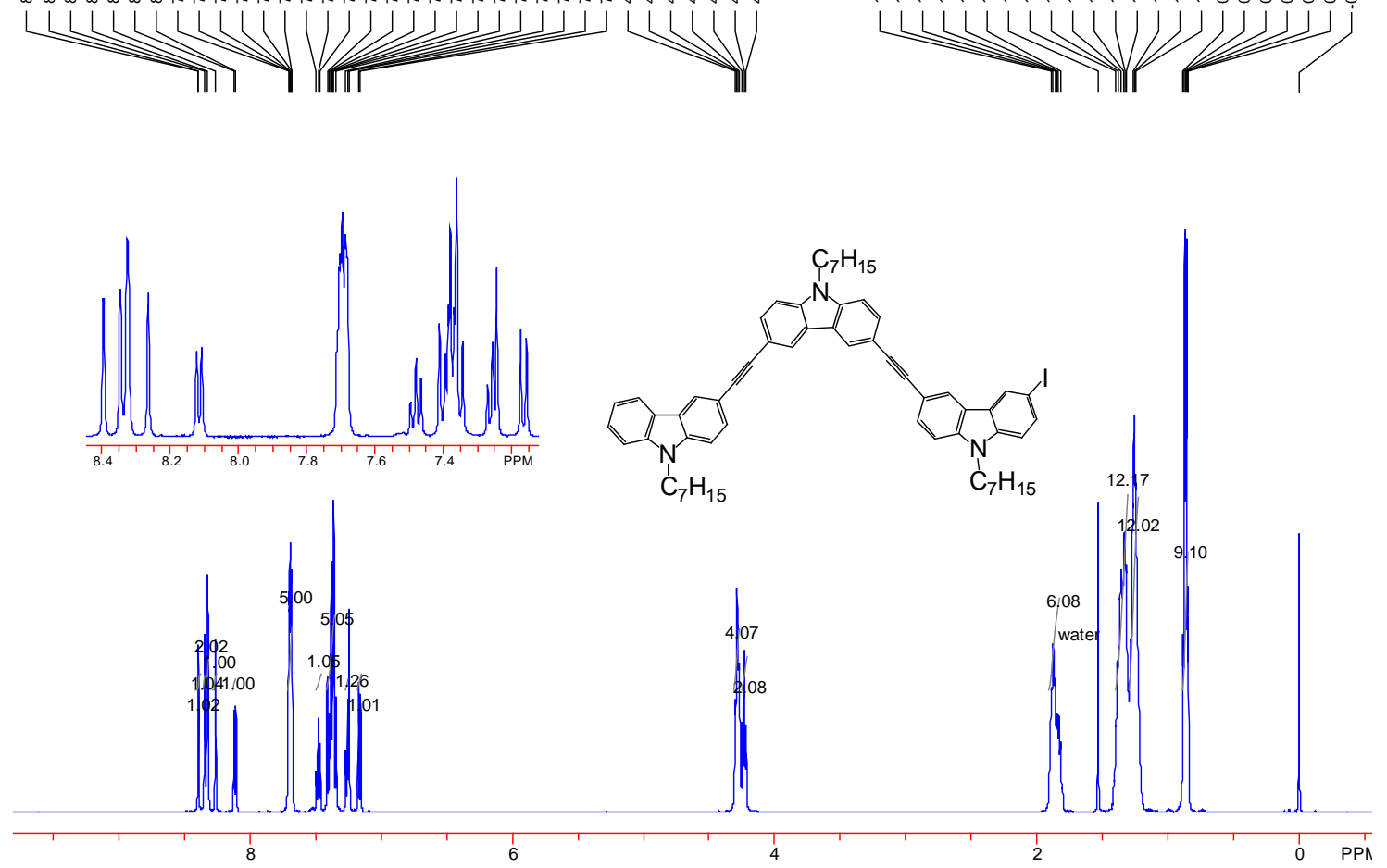

Figure S16: ${ }^{1} \mathrm{H}$ NMR spectrum of compound $\mathbf{1 0}$. 

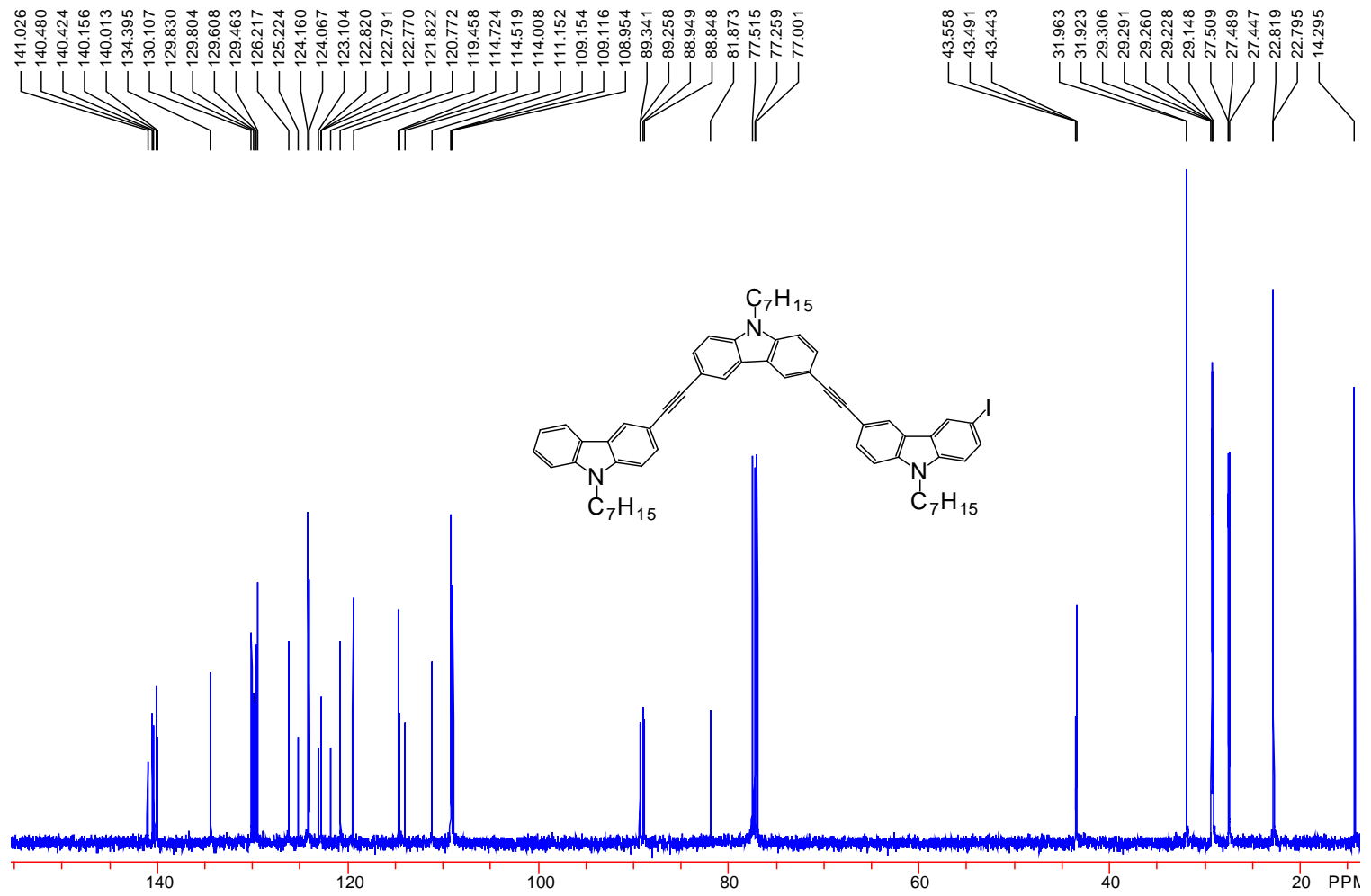

Figure S17: ${ }^{1} \mathrm{H}$ NMR spectrum of compound $\mathbf{1 0}$.

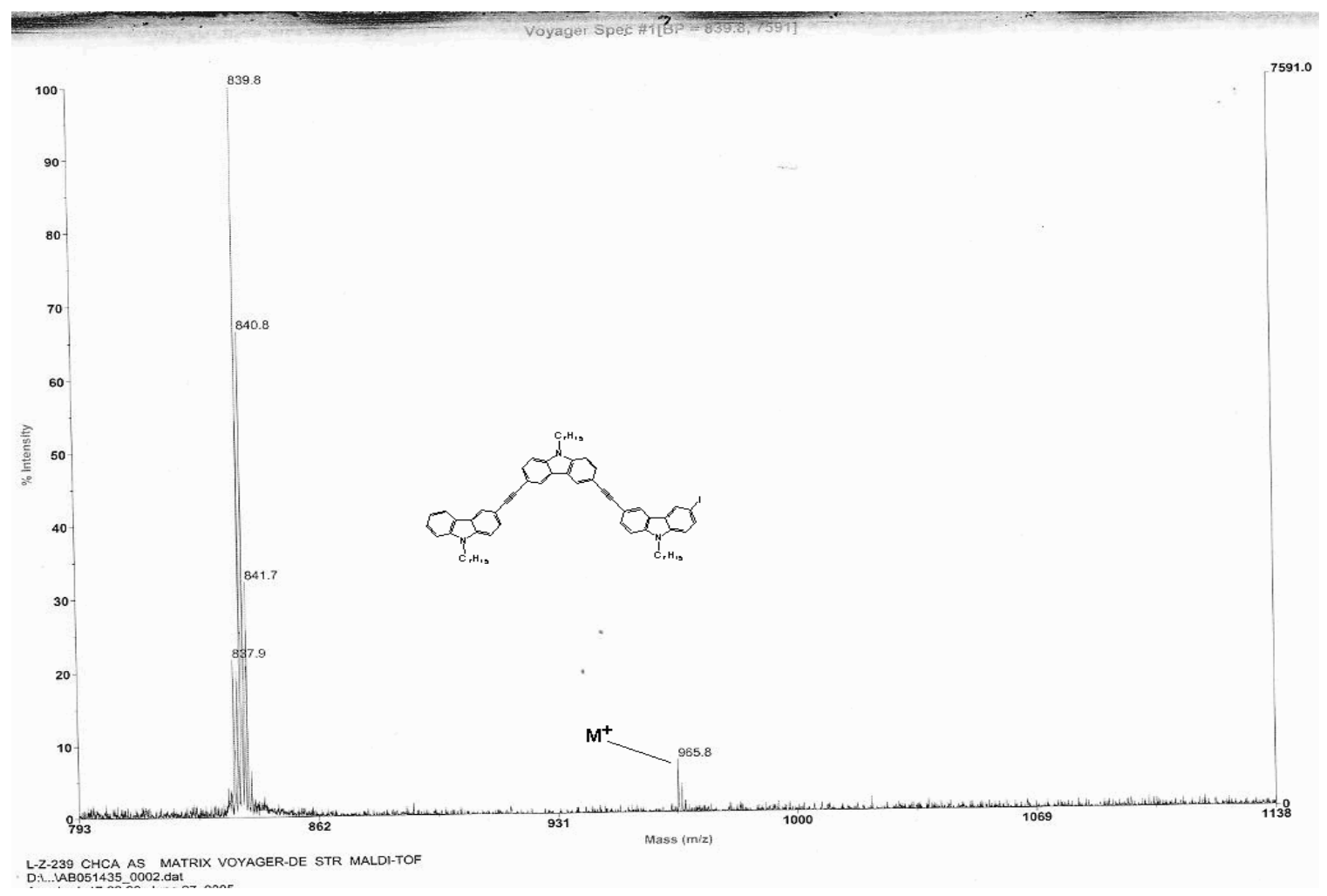

Figure S18: ${ }^{13} \mathrm{C}$ NMR spectrum of compound 10. 

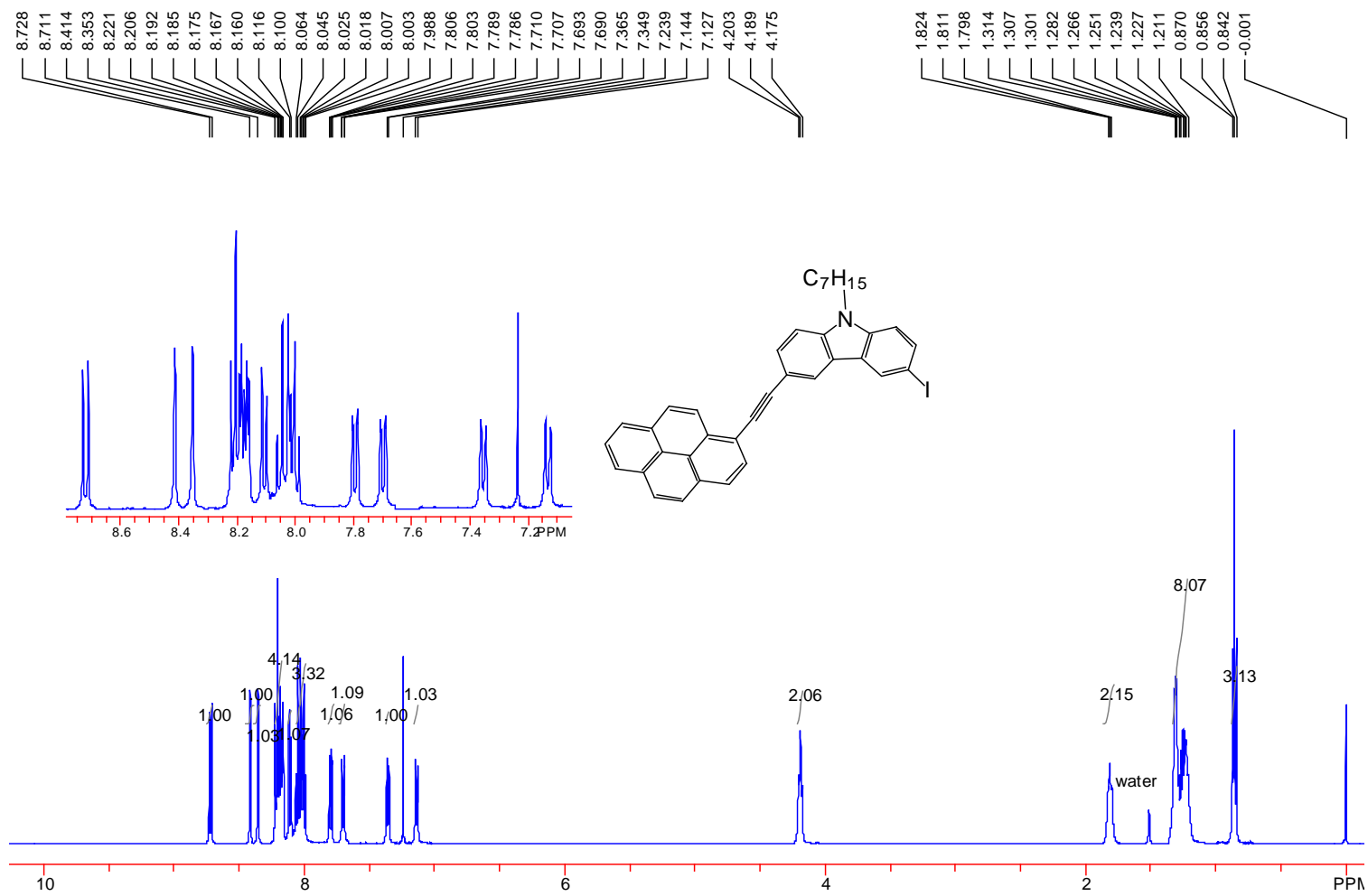

Figure S19: ${ }^{1} \mathrm{H}$ NMR spectrum of compound 11.

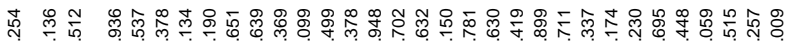

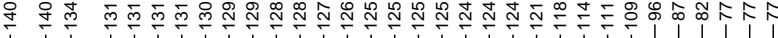

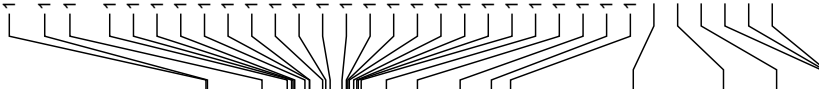
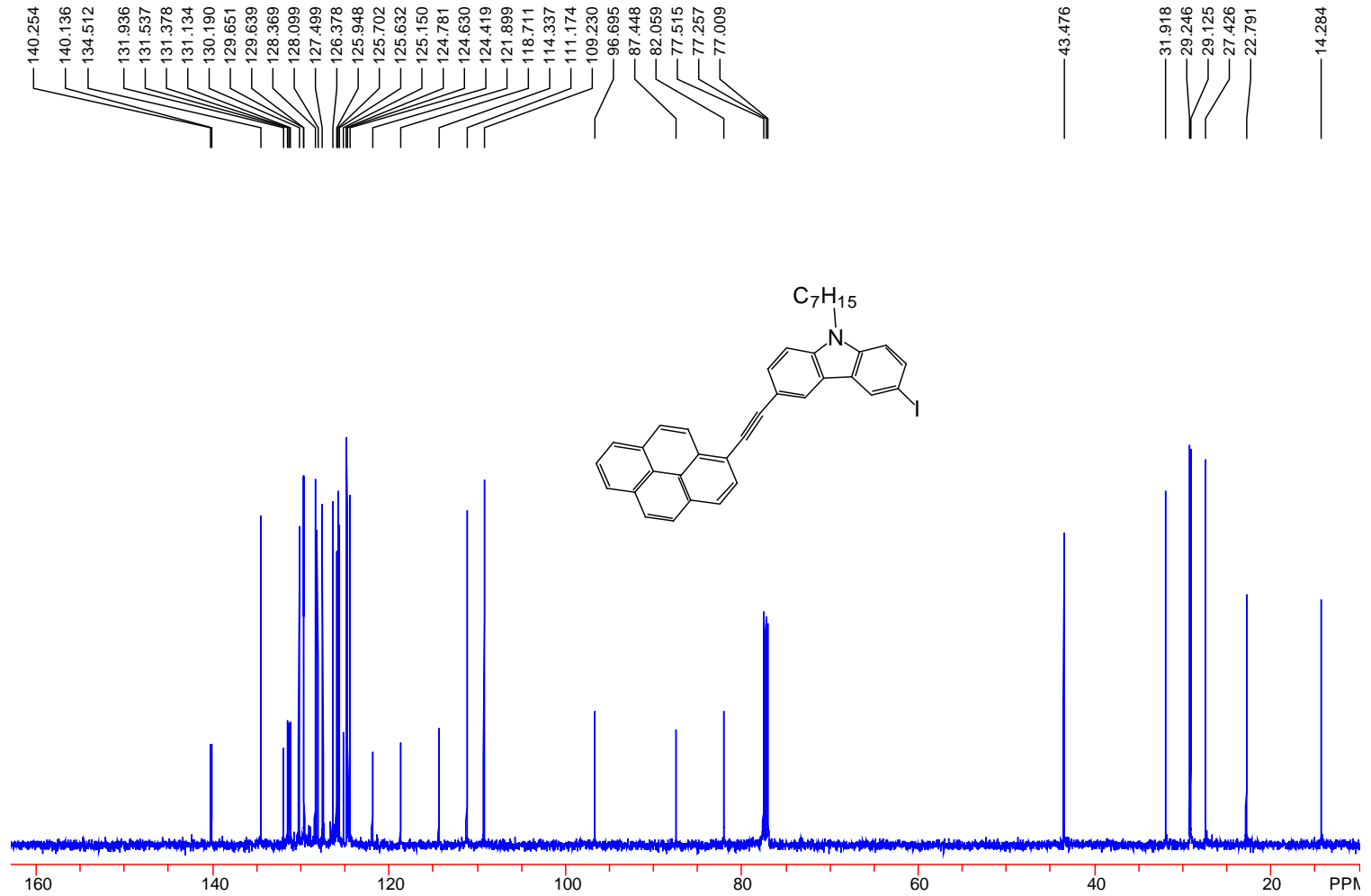

Figure S20: ${ }^{13} \mathrm{C}$ NMR spectrum of compound $\mathbf{1 1}$. 


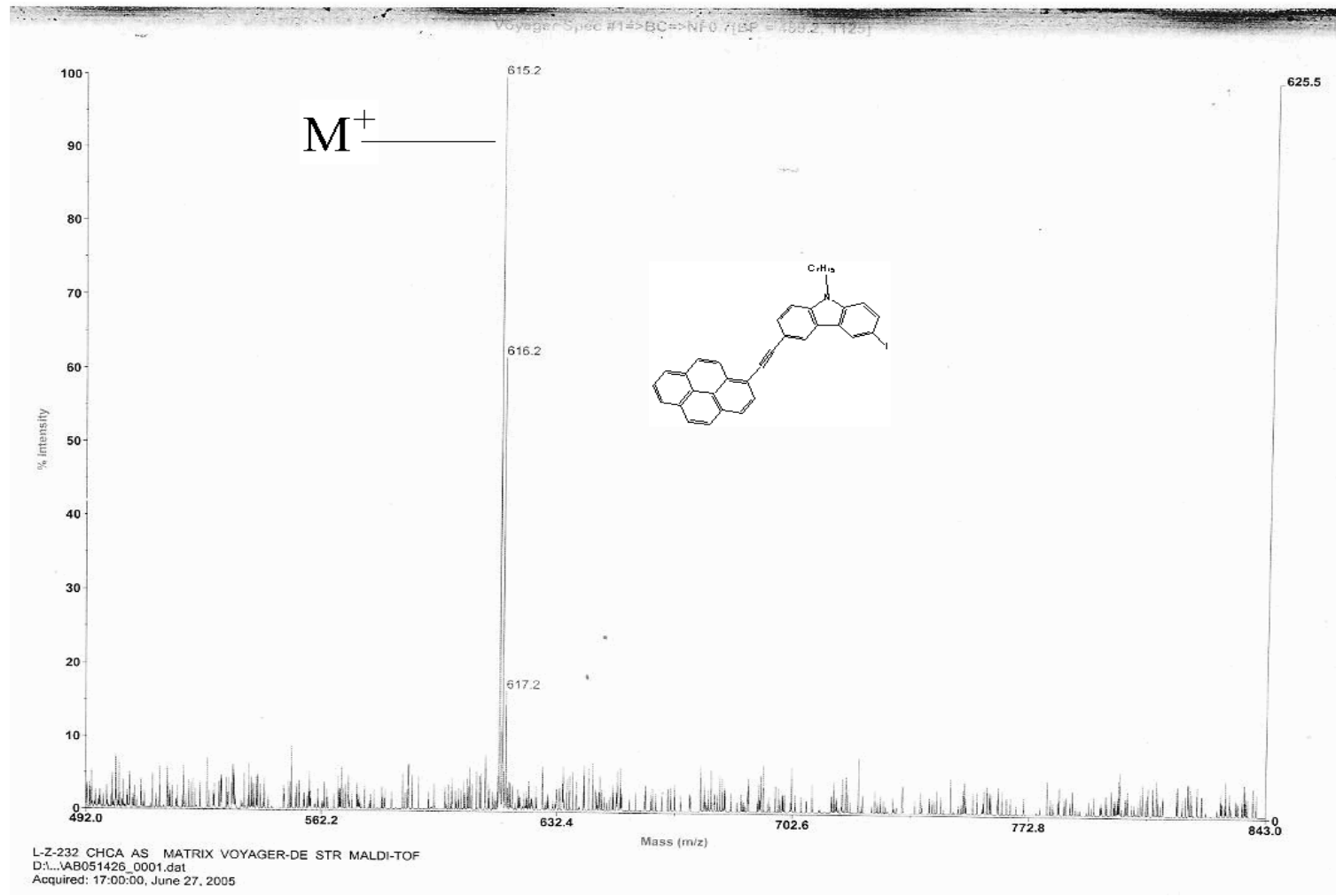

Figure S21: mass spectrum of compound 11.
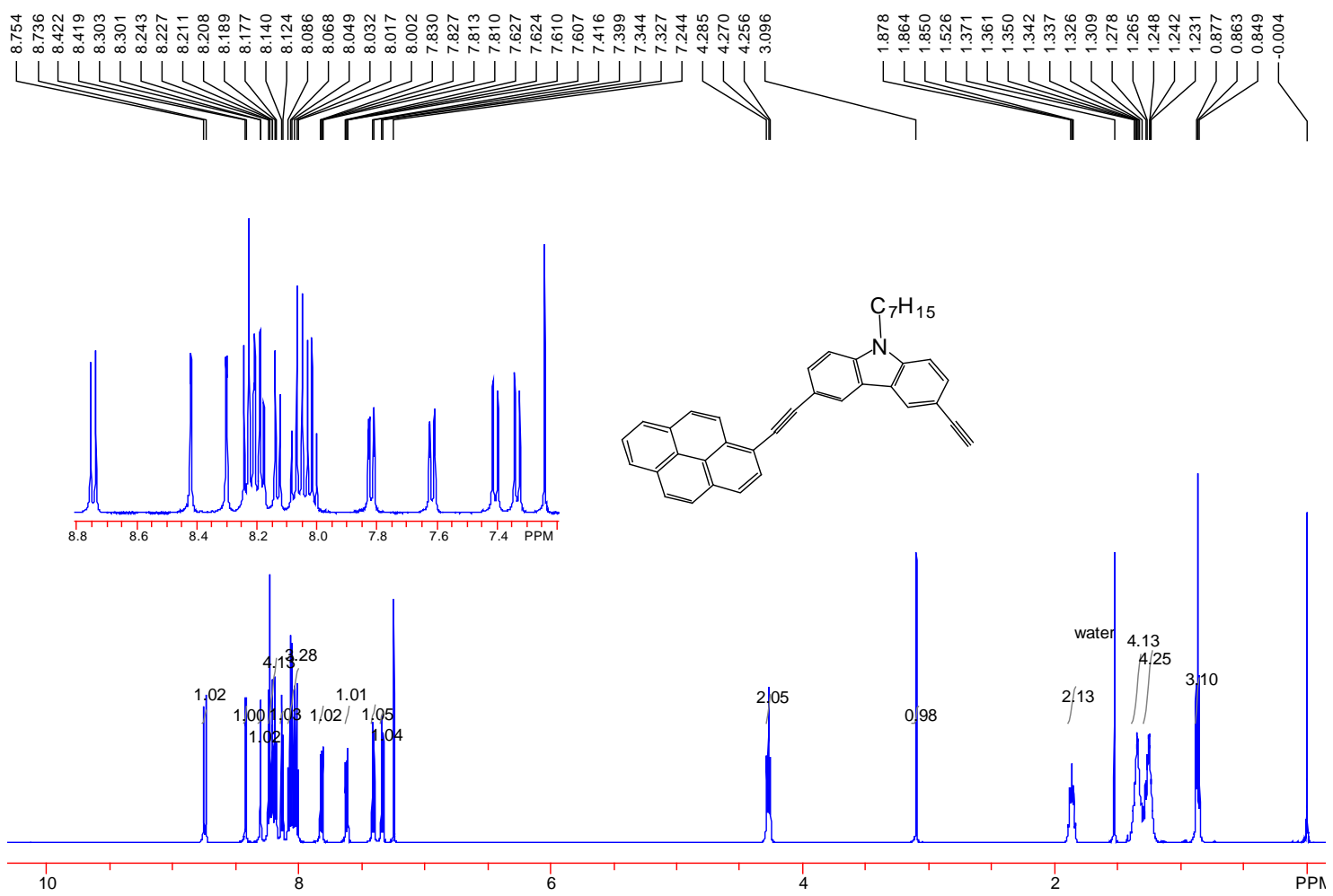

Figure S22: ${ }^{1} \mathrm{H}$ NMR spectrum of compound 13. 

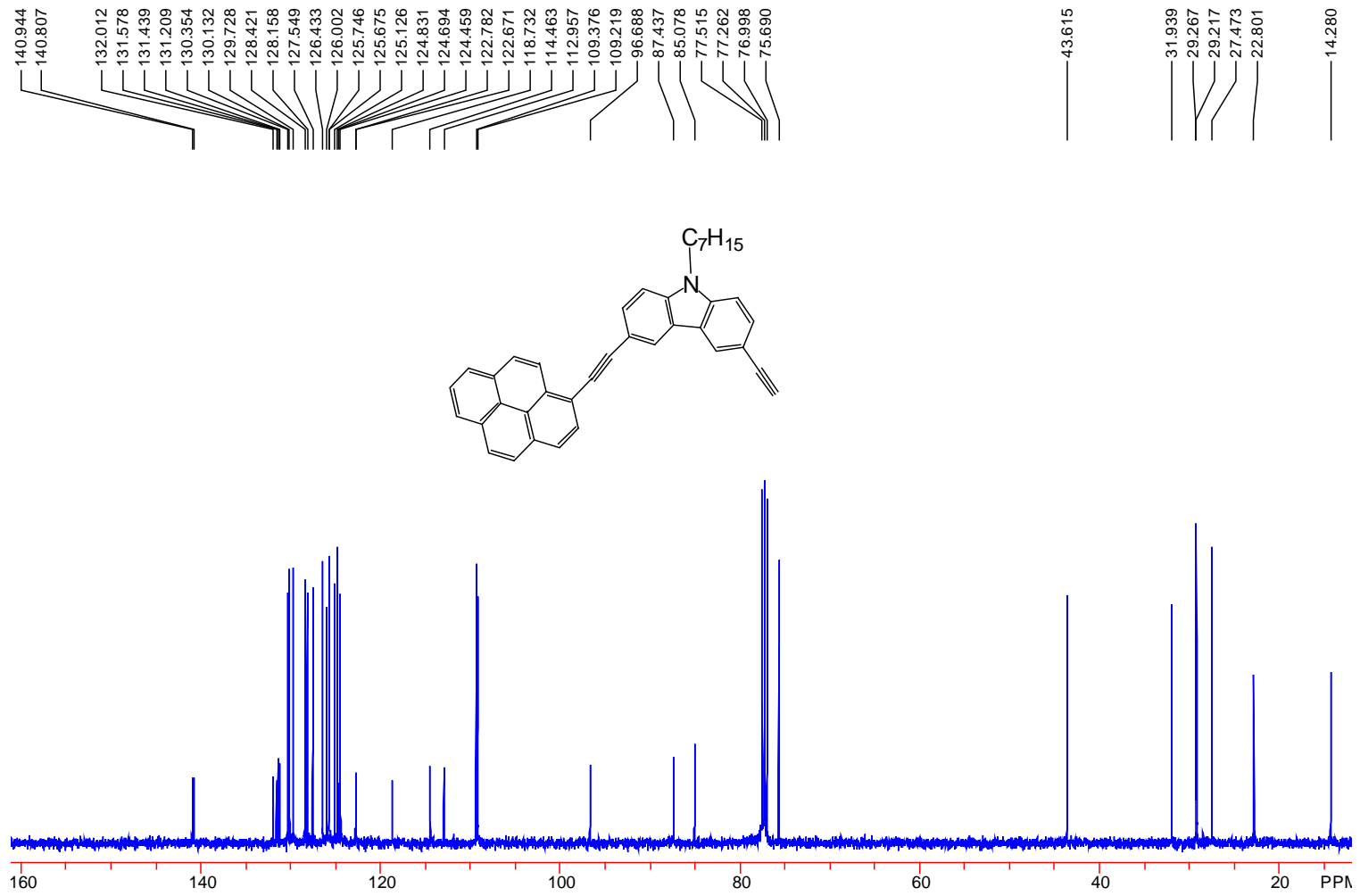

Figure S23: ${ }^{13} \mathrm{C}$ NMR spectrum of compound 13.

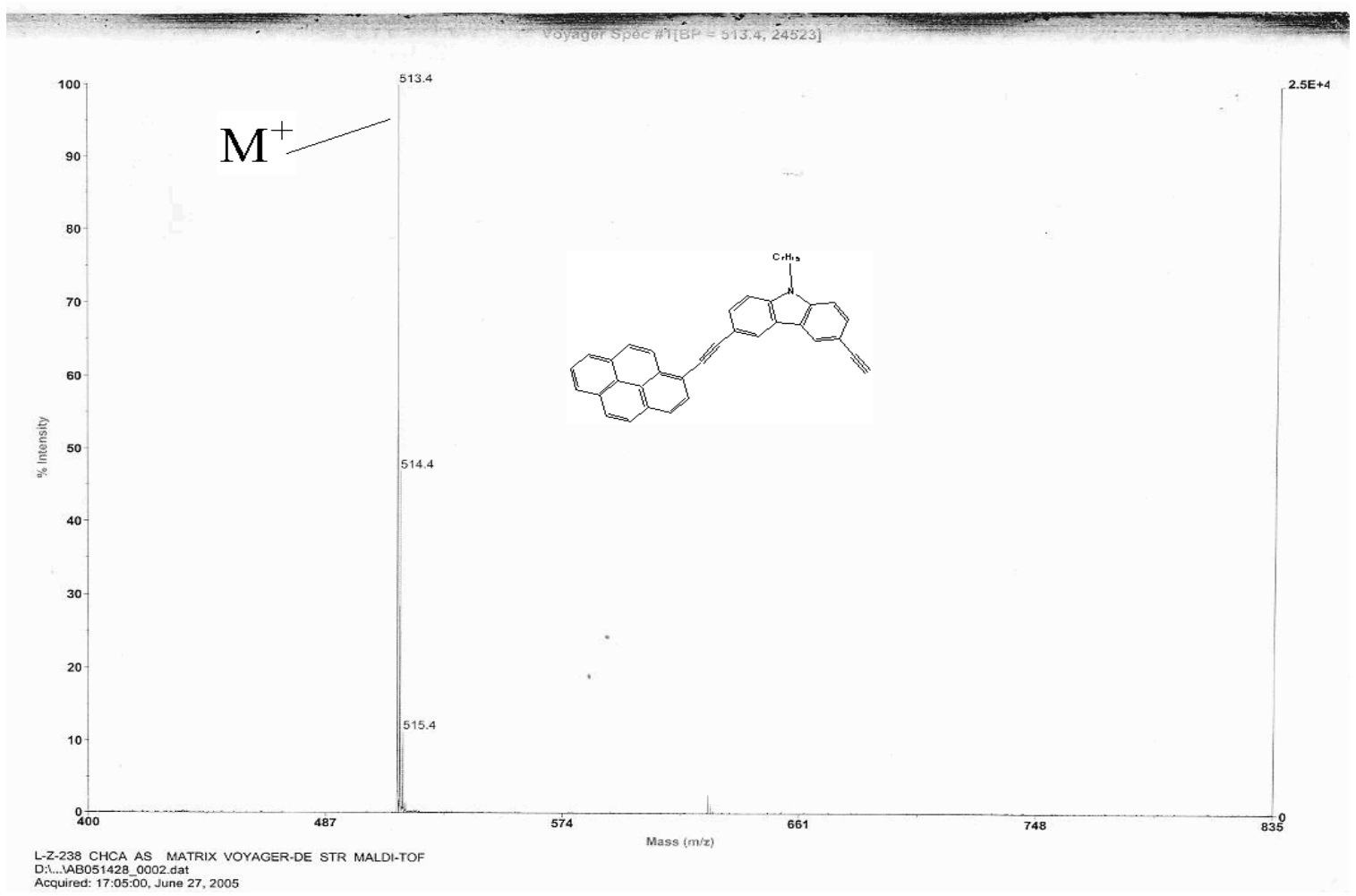

Figure S24: mass spectrum of compound 13. 

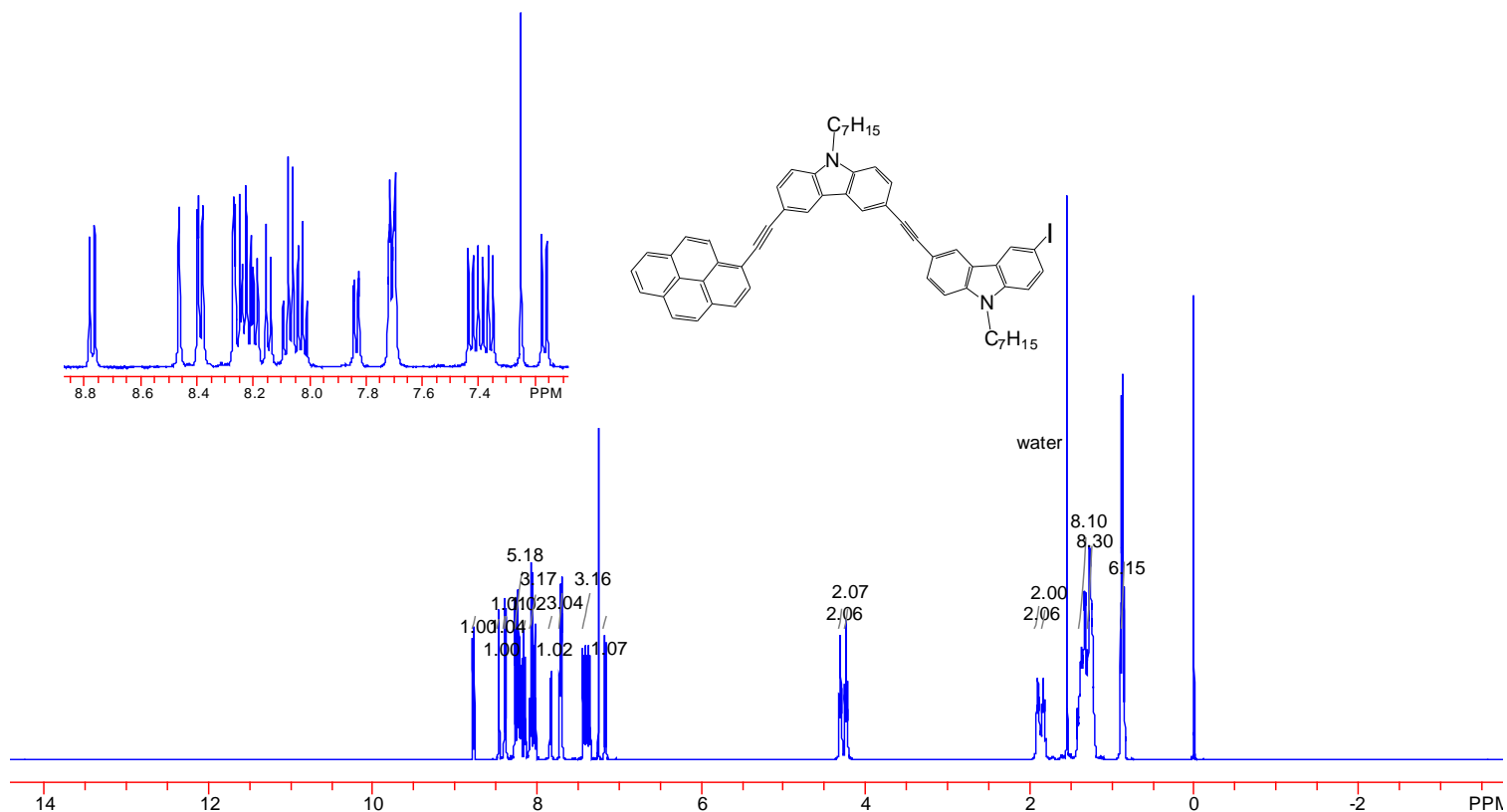

Figure S25: ${ }^{1} \mathrm{H}$ NMR spectrum of compound 14.
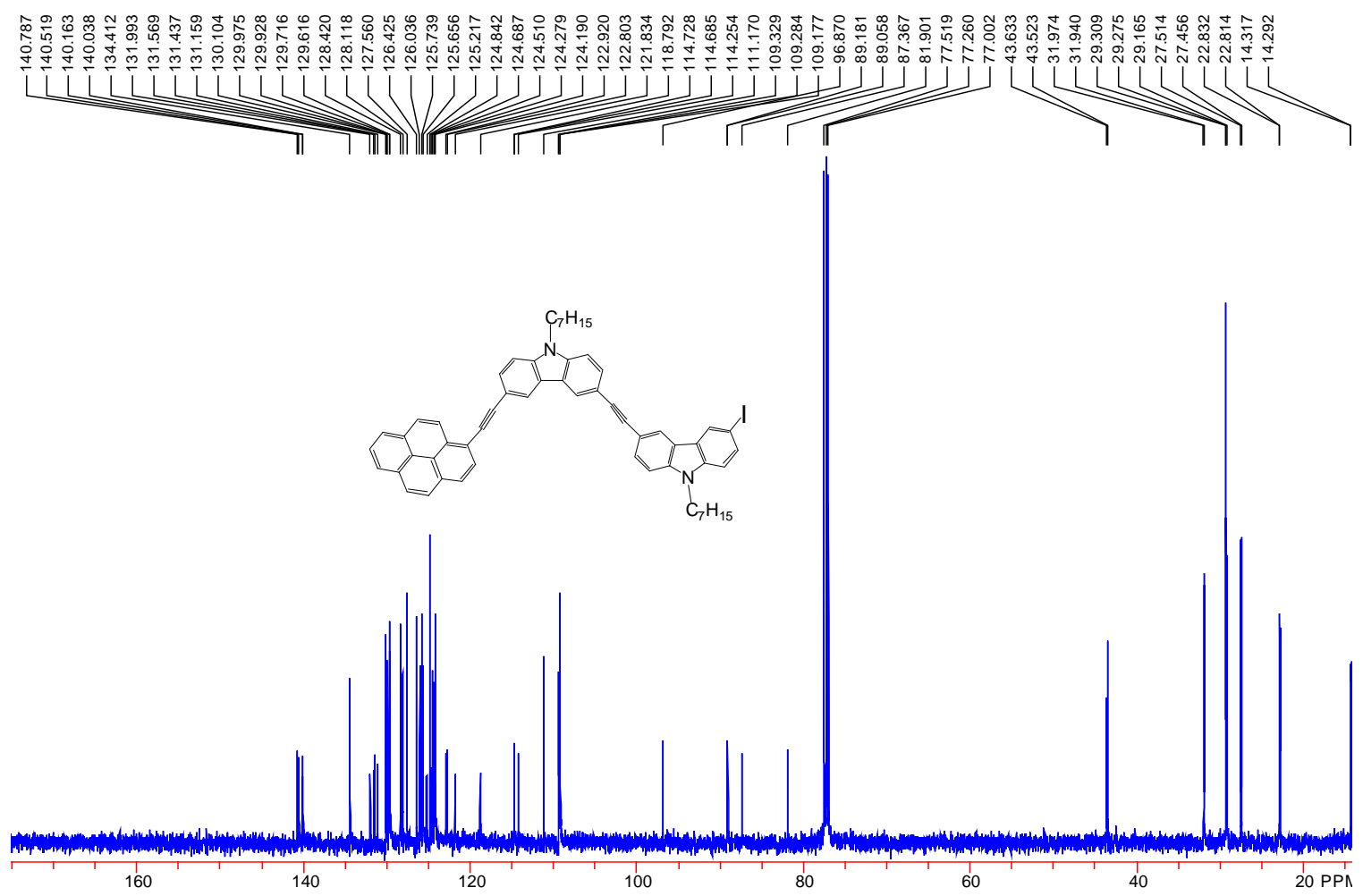

Figure S26: mass spectrum of compound 14. 


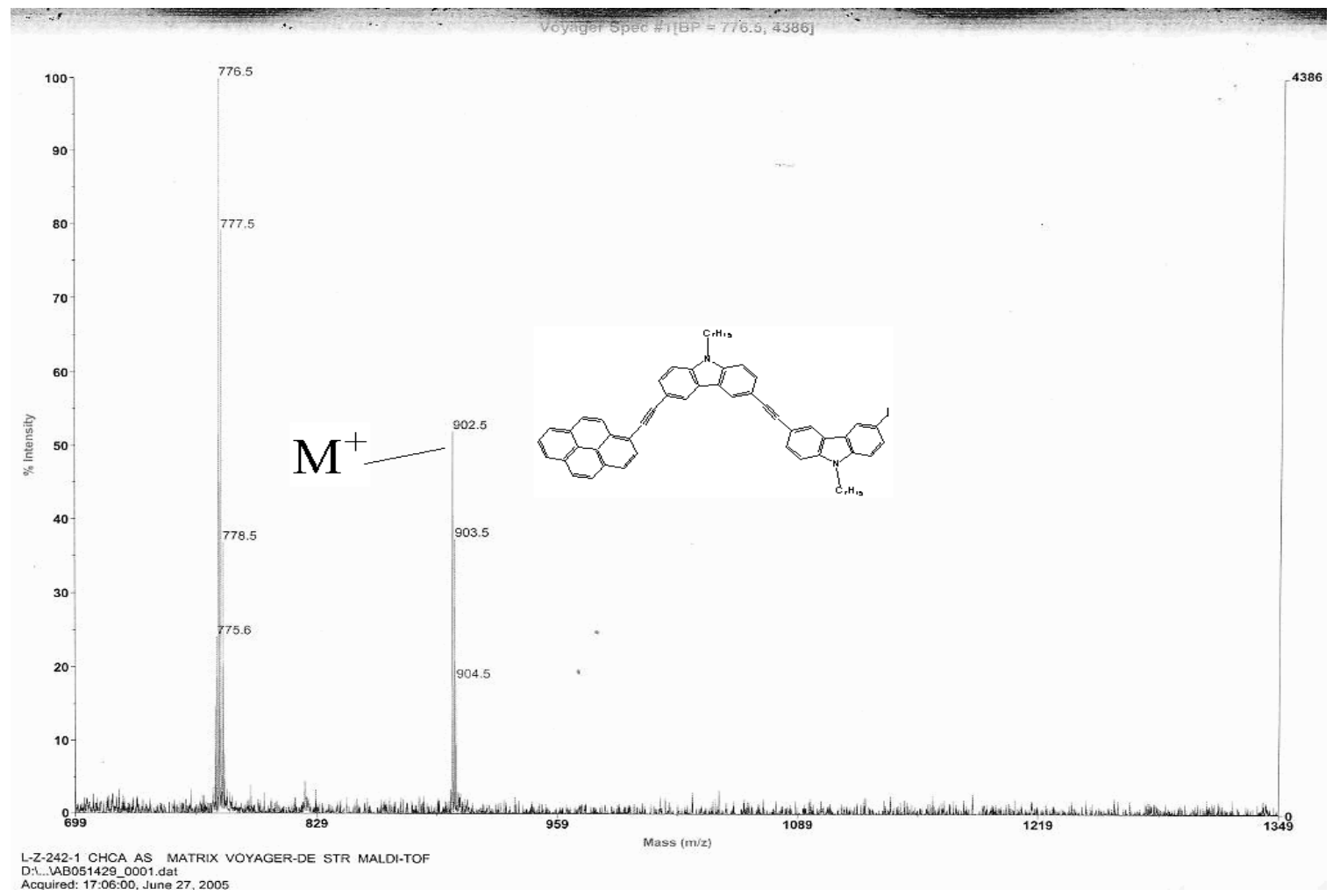

Figure S27: mass spectrum of compound 14.

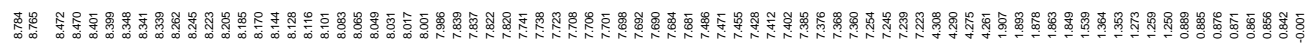
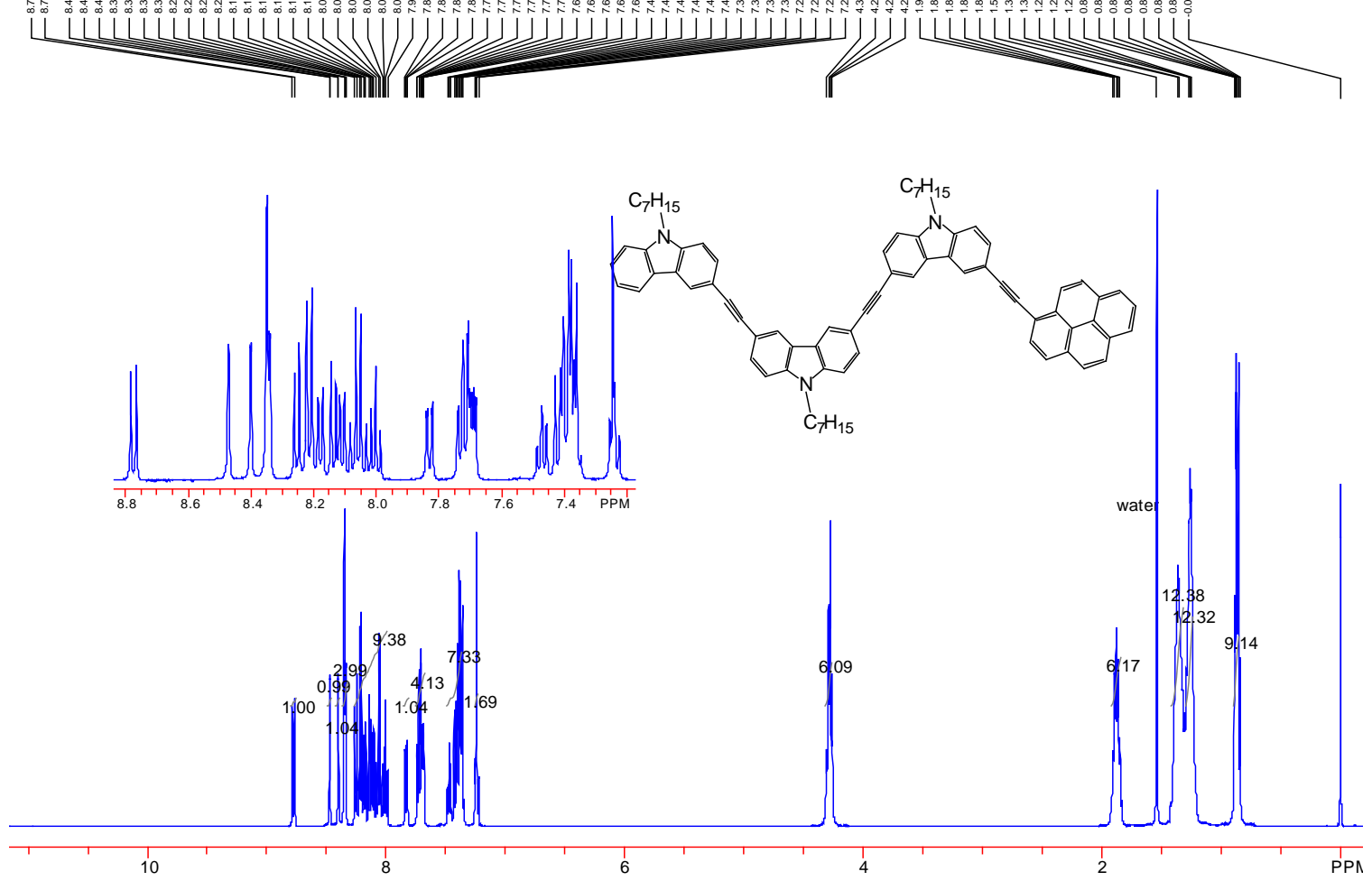

Figure S28: ${ }^{1} \mathrm{H}$ NMR spectrum of compound PyCz3. 


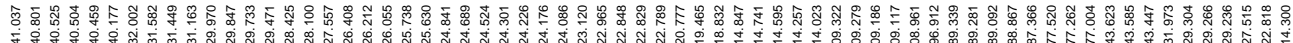

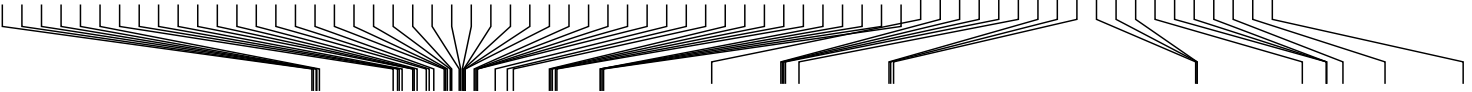

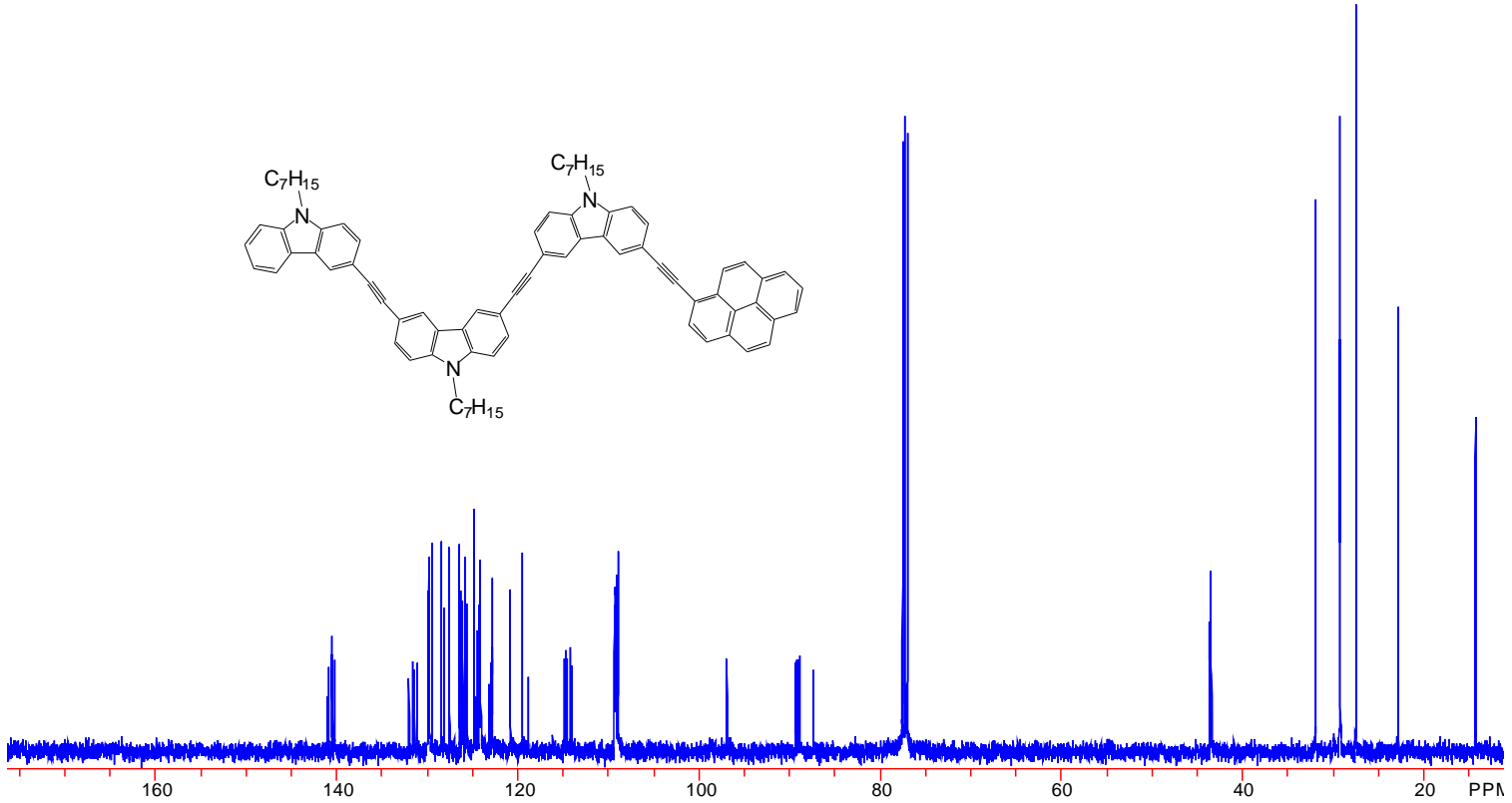

Figure S29: ${ }^{13} \mathrm{C}$ NMR spectrum of compound PyCz3.

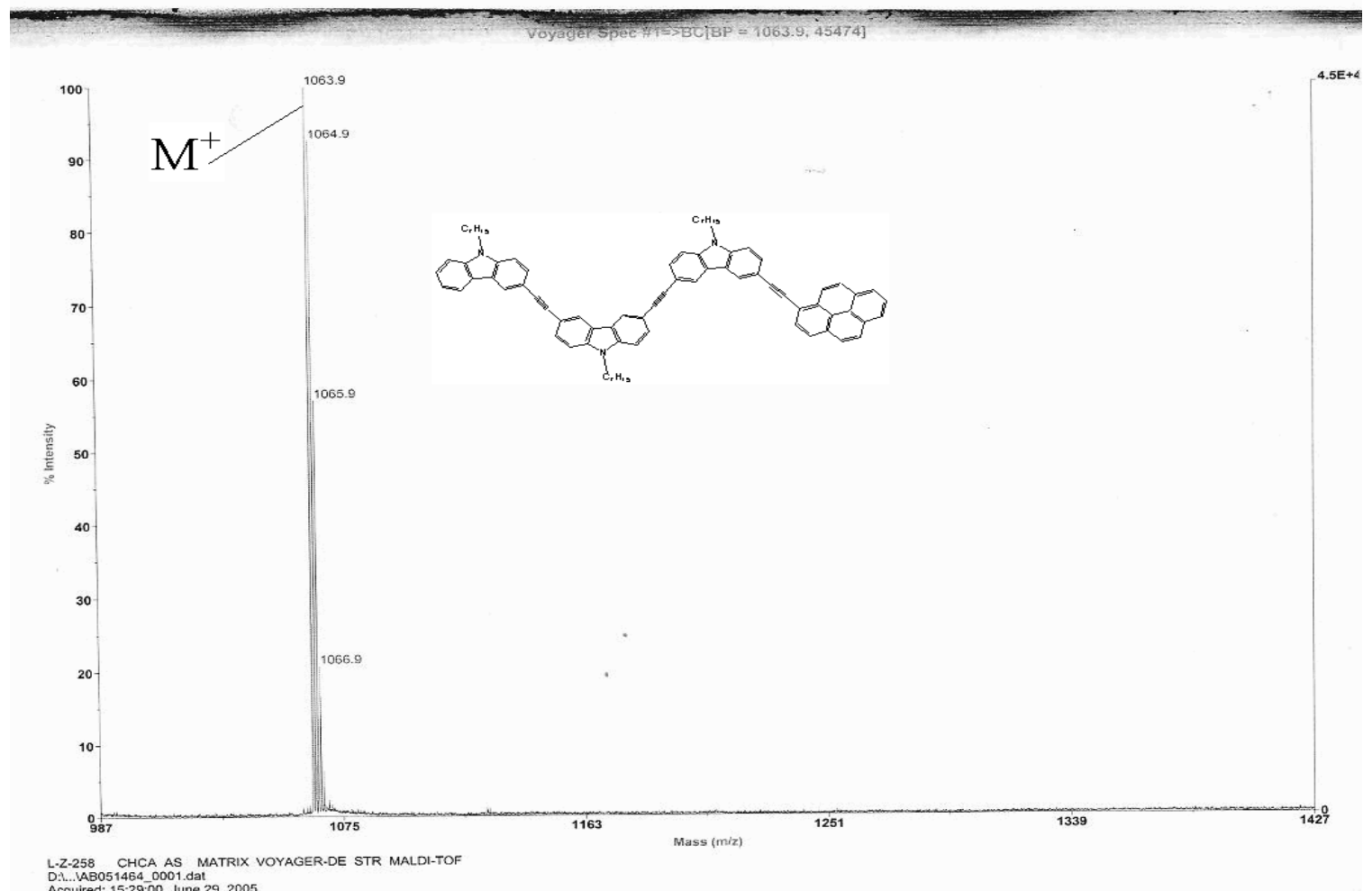

Figure S30: mass spectrum of compound PyCz3. 


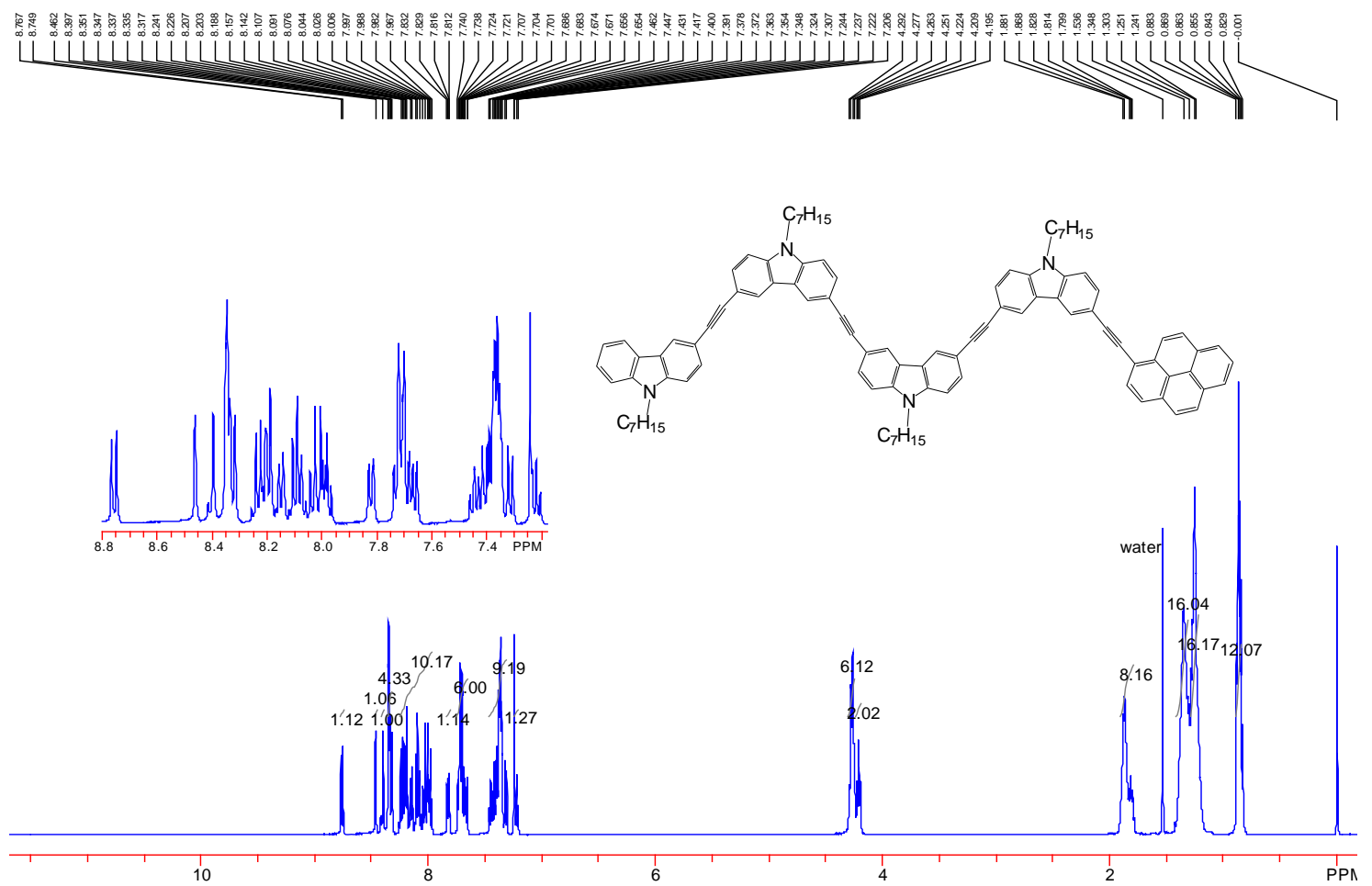

Figure S31: ${ }^{1} \mathrm{H}$ NMR spectrum of compound PyCz4.
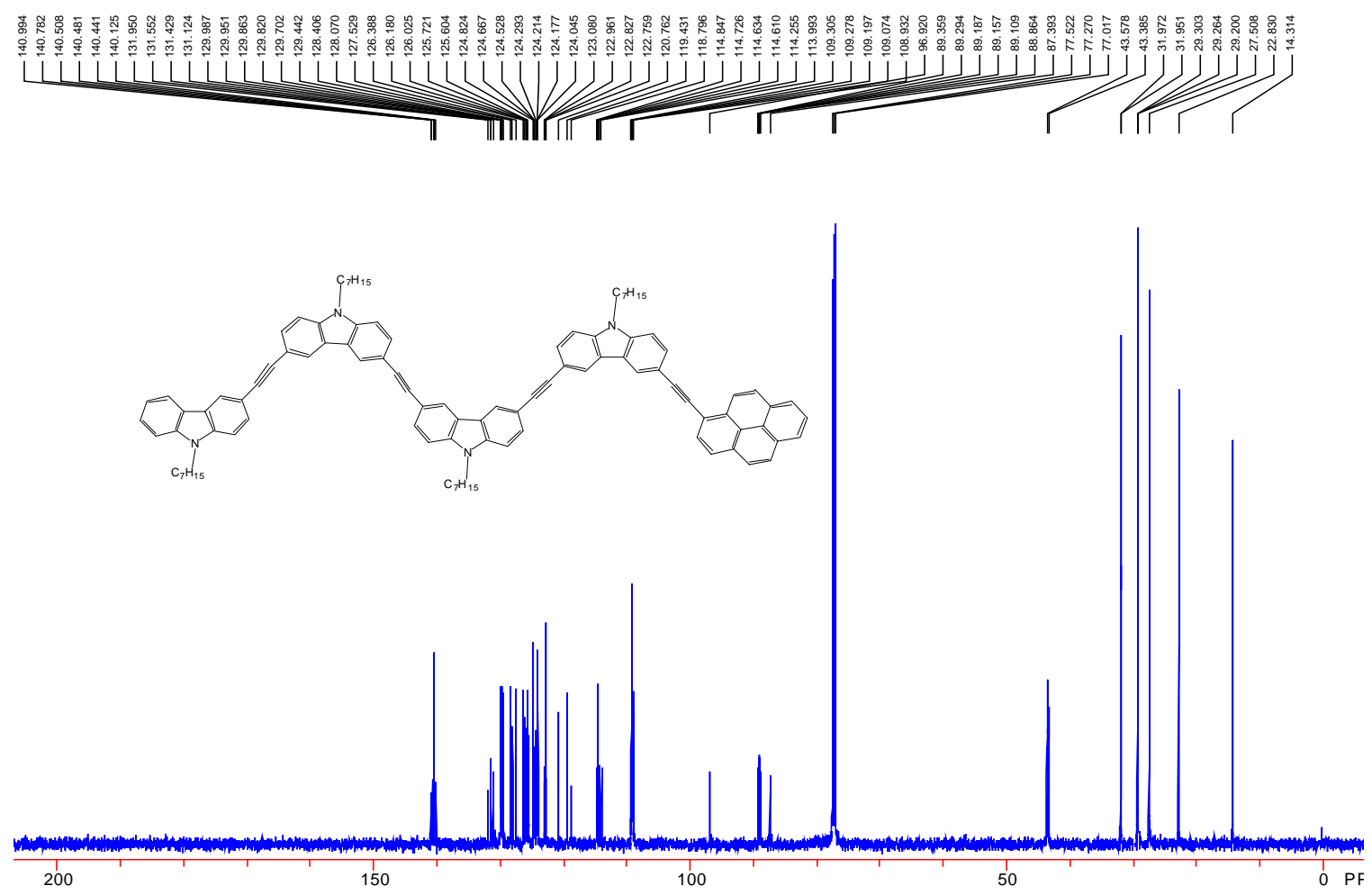

Figure S32: ${ }^{13} \mathrm{C}$ NMR spectrum of compound PyCz4. 


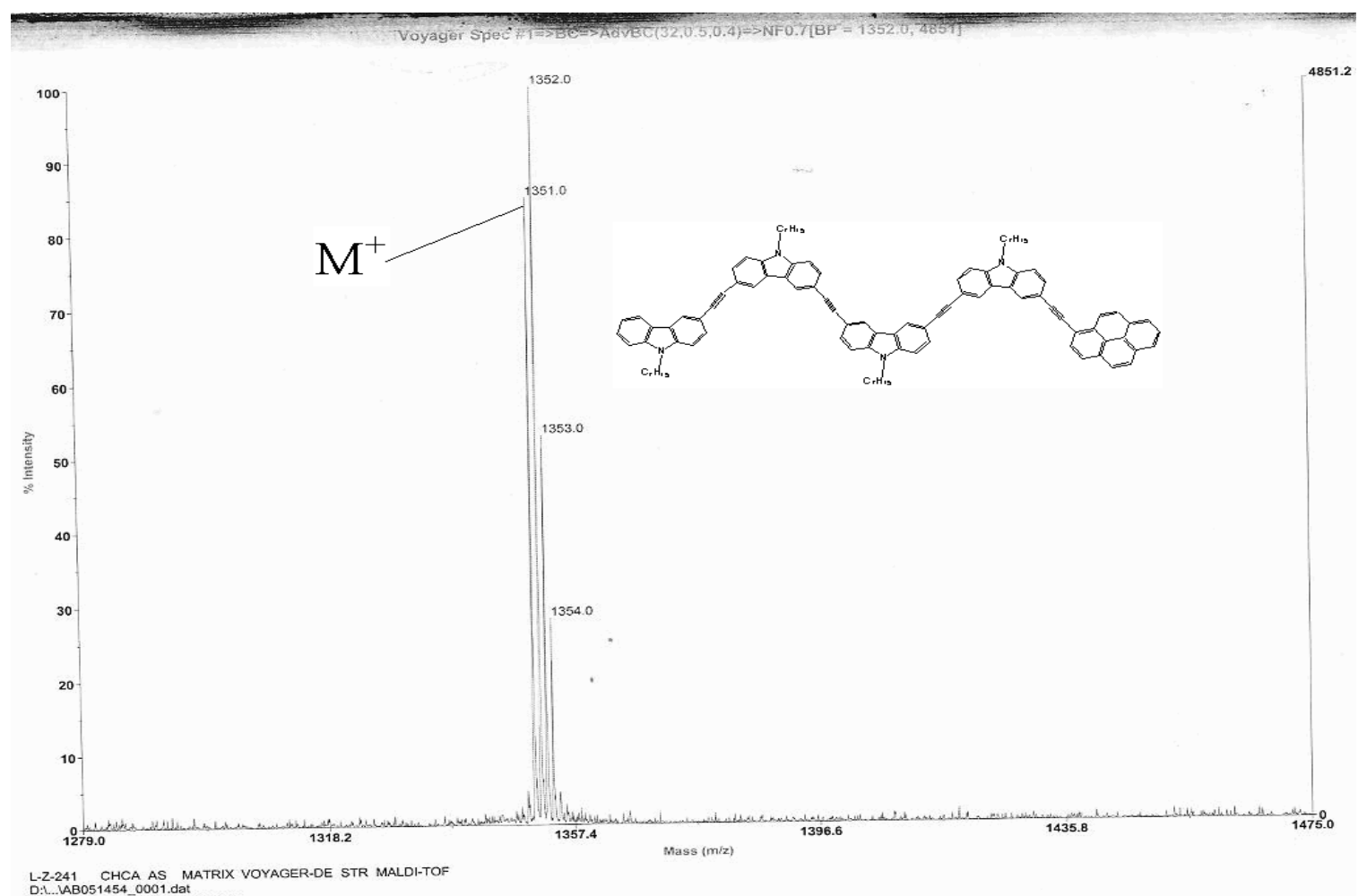

Figure S33: mass spectrum of compound PyCz4.
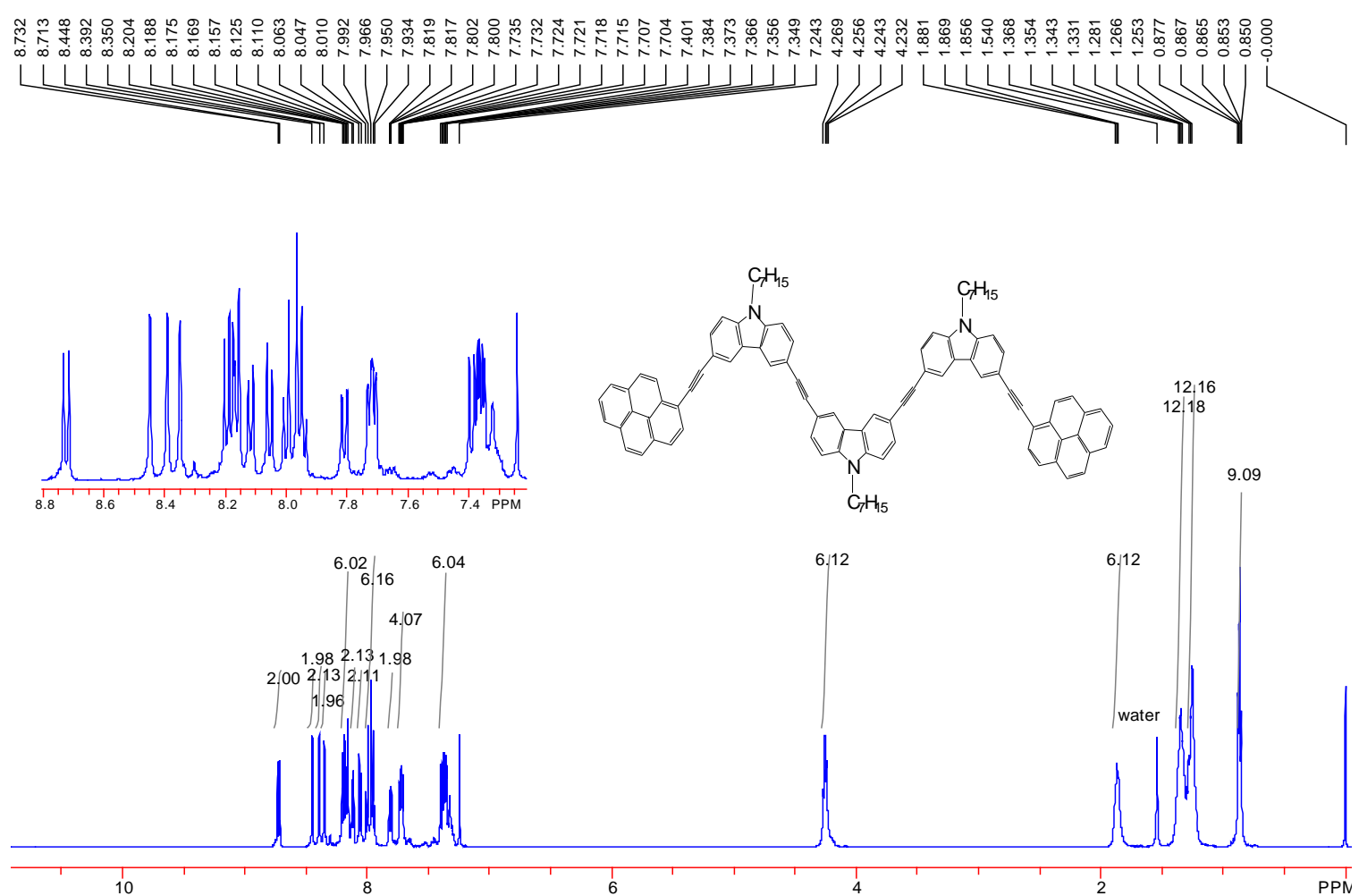

Figure S34: ${ }^{1} \mathrm{H}$ NMR spectrum of compound PyCz3Py. 

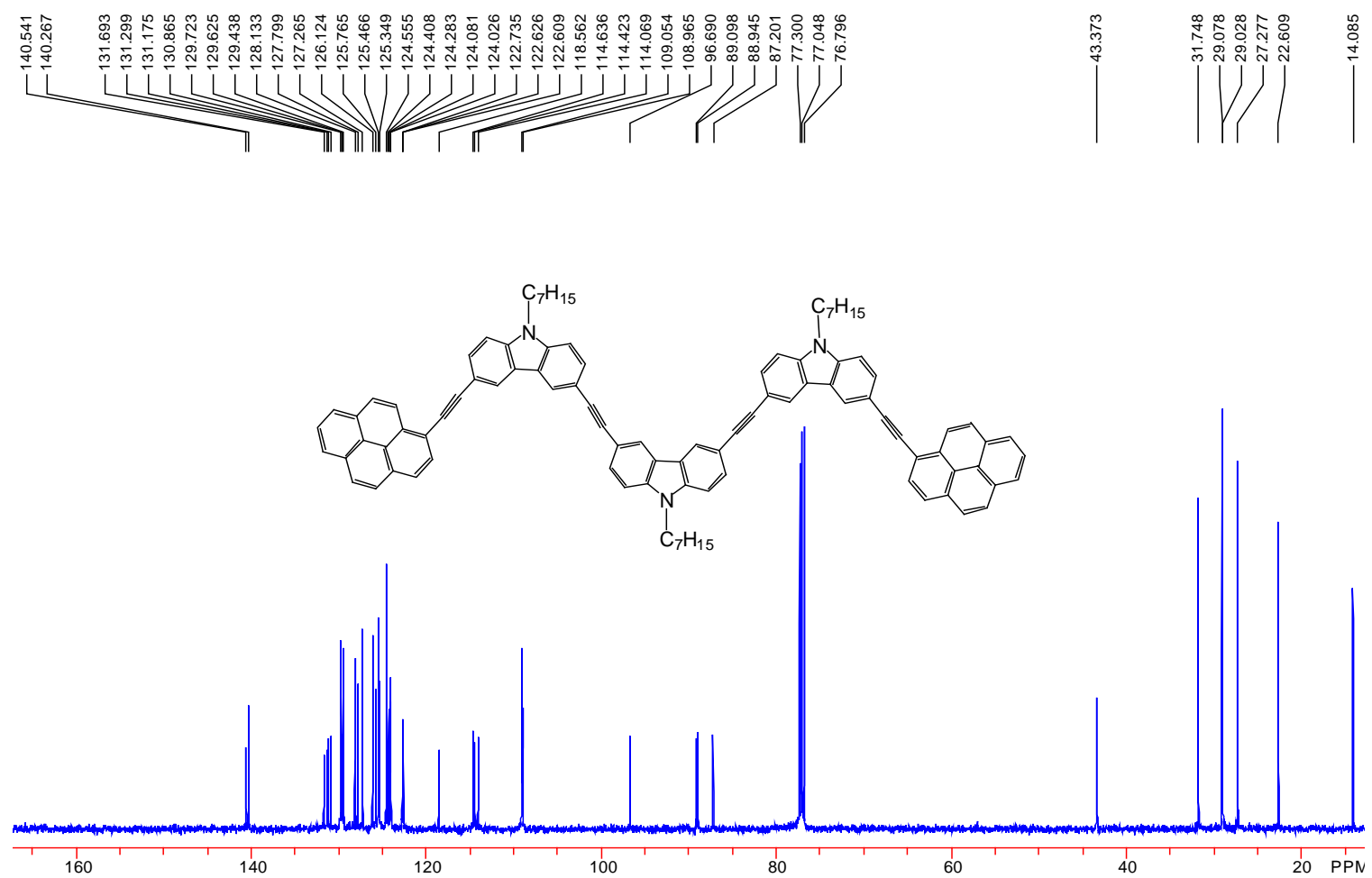

Figure S35: ${ }^{13} \mathrm{C}$ NMR spectrum of compound PyCz3Py.

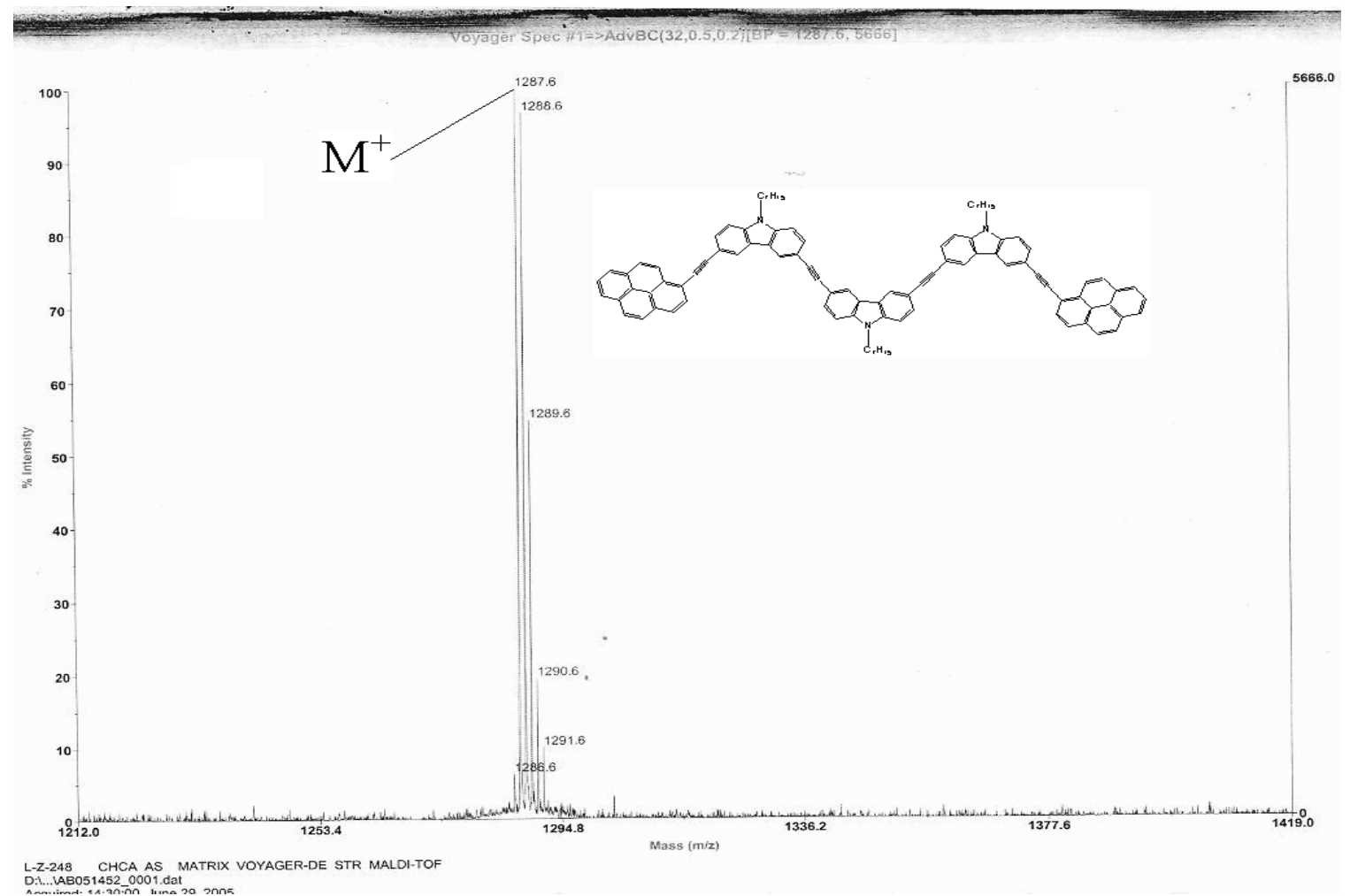

Figure S36: mass spectrum of compound PyCz3Py. 

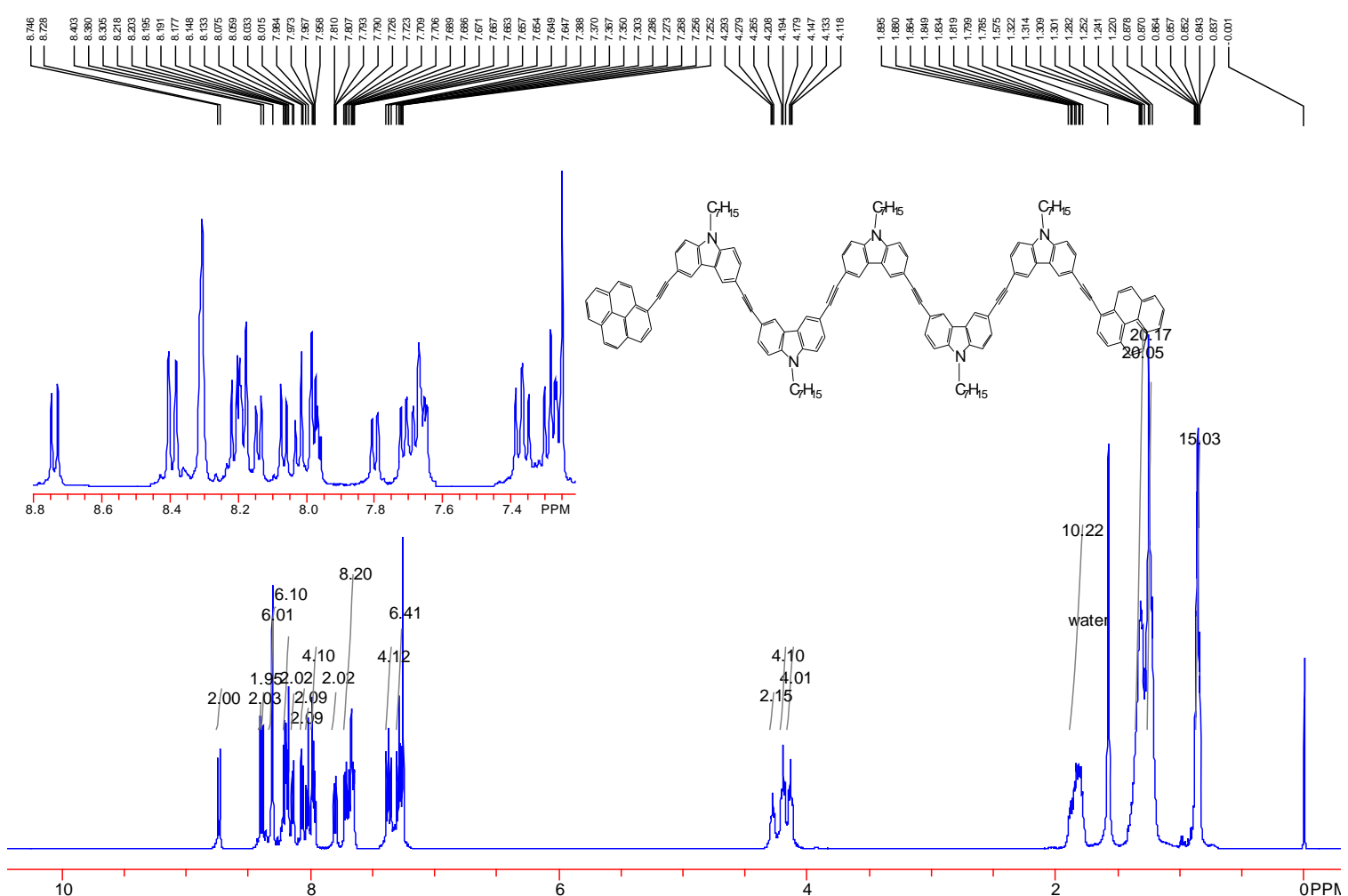

Figure S37: ${ }^{1} \mathrm{H}$ NMR spectrum of compound PyCz5Py.
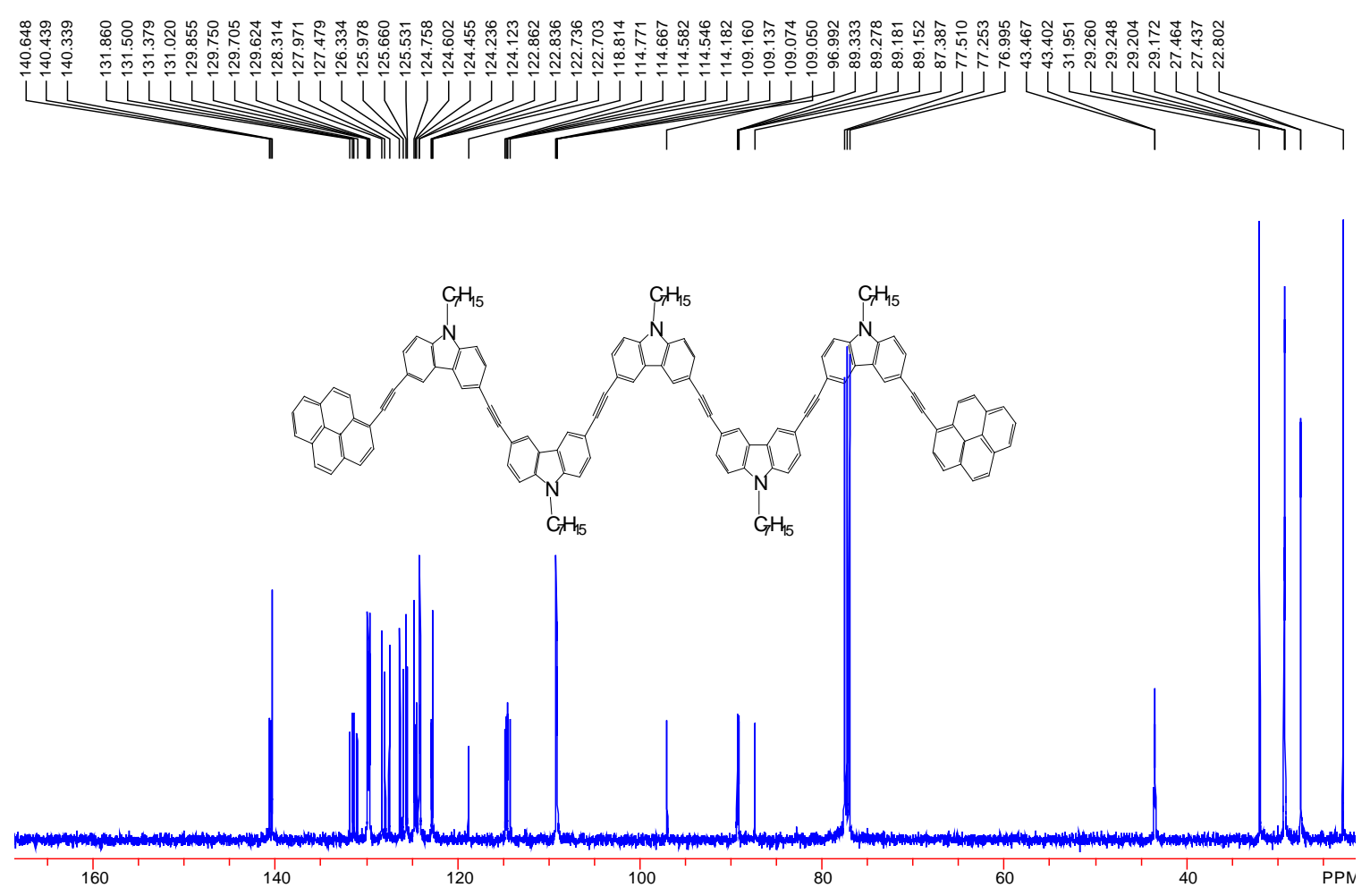

Figure S38: ${ }^{13} \mathrm{C}$ NMR spectrum of compound PyCz5Py. 


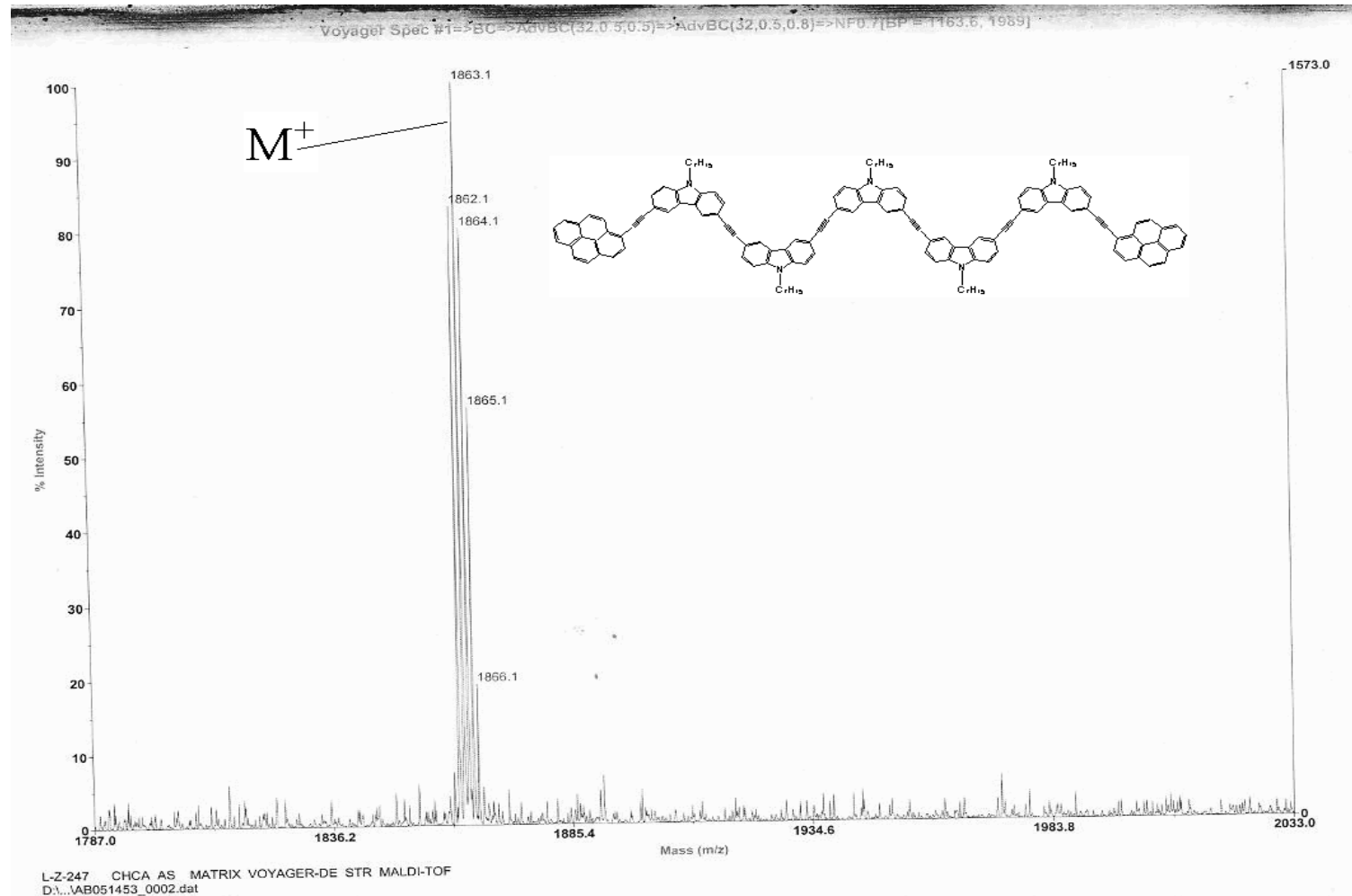

Figure S39: mass spectrum of compound PyCz5Py.

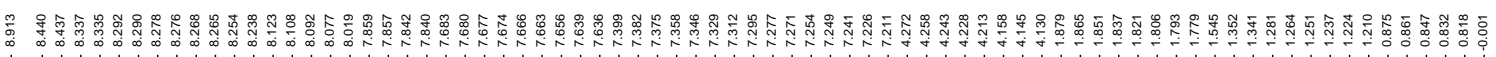
LัLLLLLLLLLLLLLUU| IIIIT

11
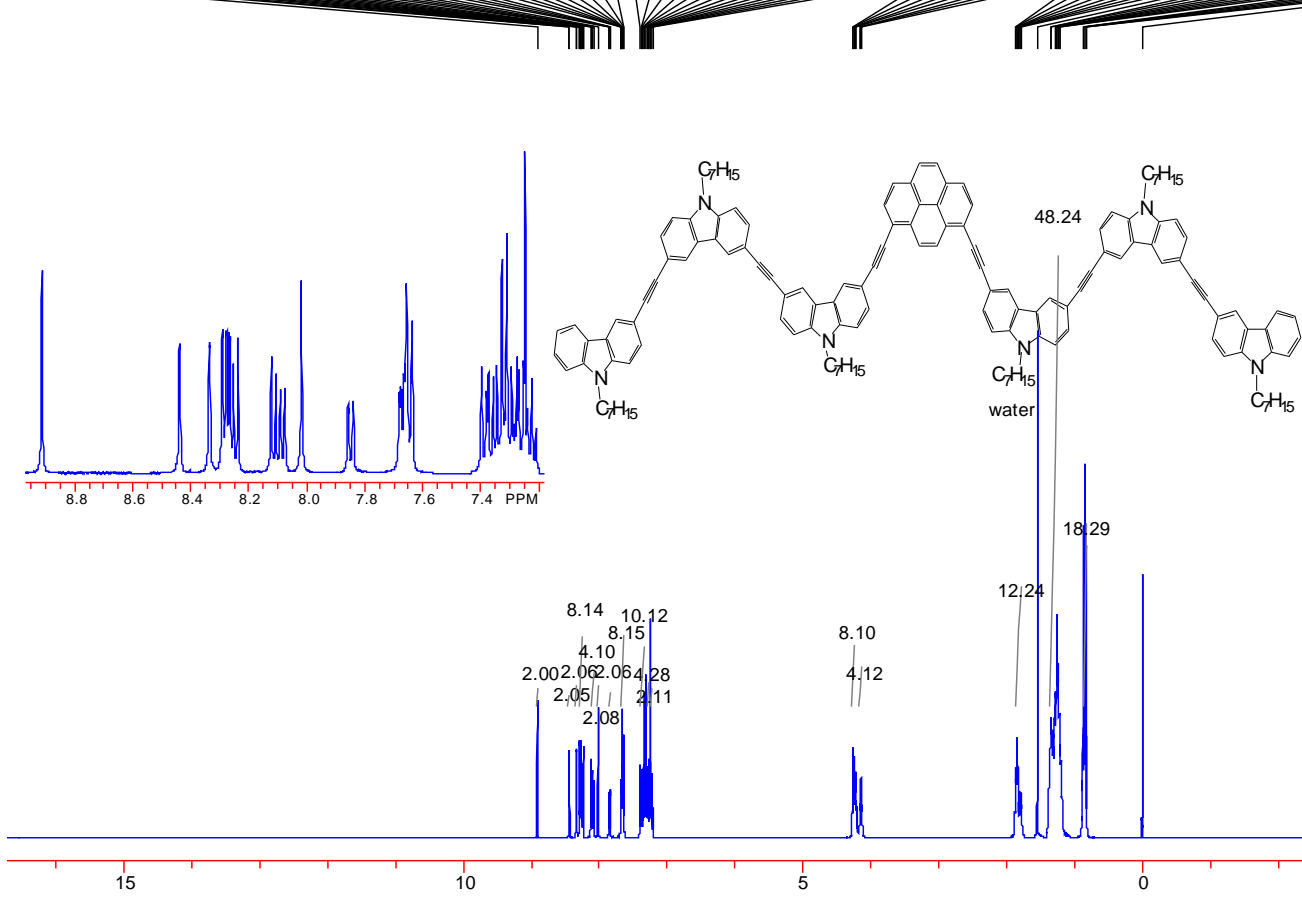

Figure S40: ${ }^{1} \mathrm{H}$ NMR spectrum of compound Cz3PyCz3. 


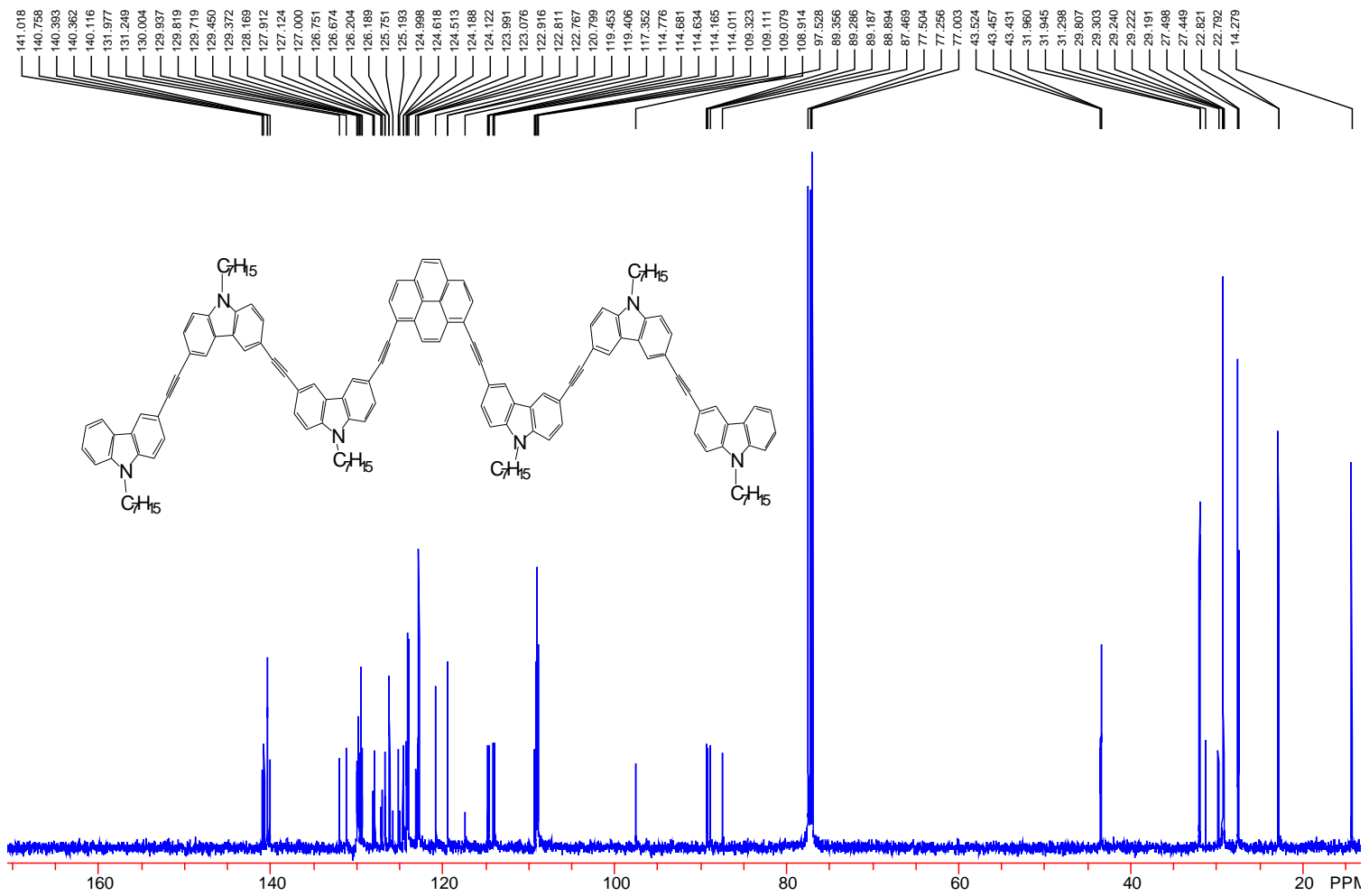

Figure S41: ${ }^{13} \mathrm{C}$ NMR spectrum of compound Cz3PyCz3.

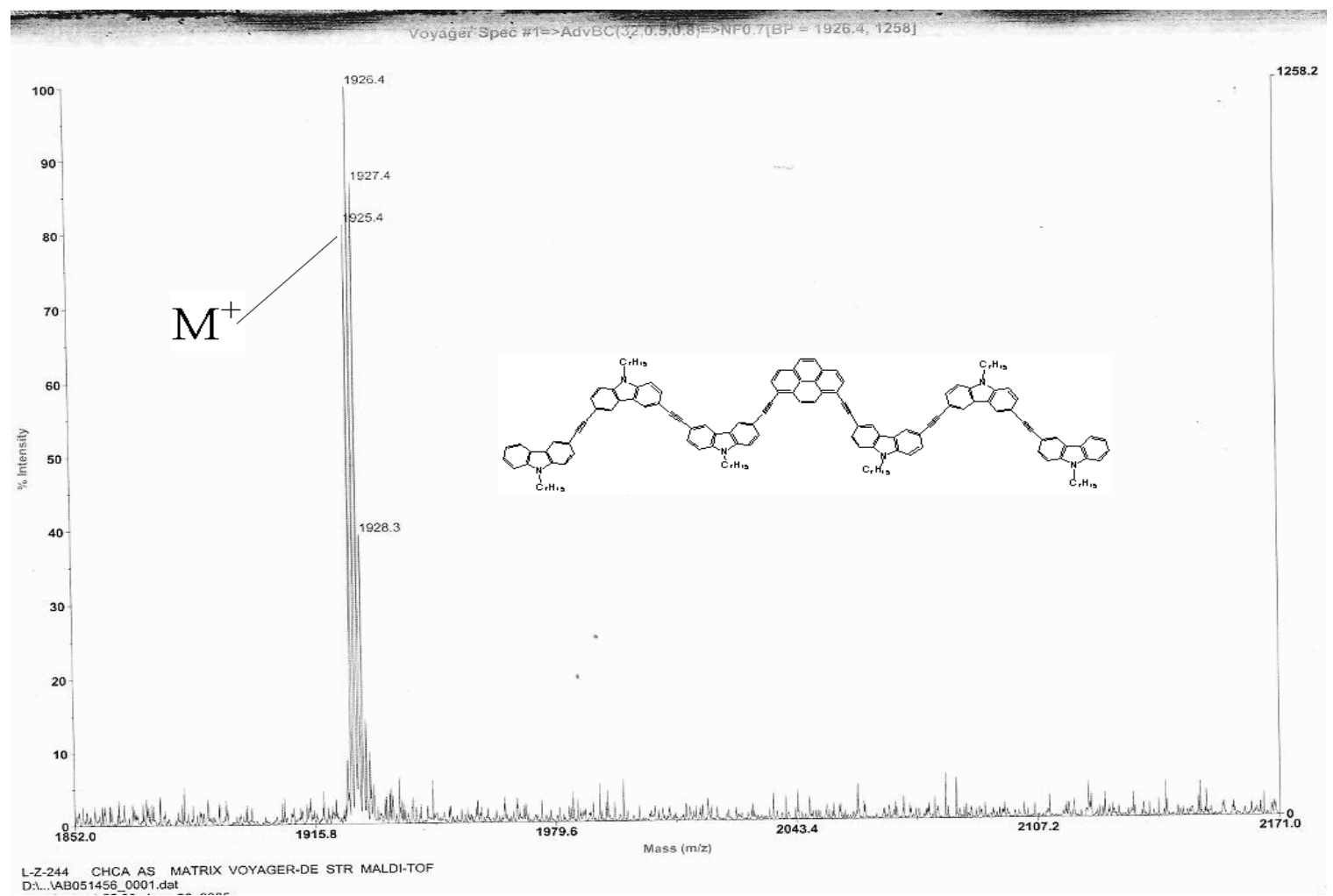

Figure S42: ${ }^{13} \mathrm{C}$ NMR spectrum of compound Cz3PyCz3. 

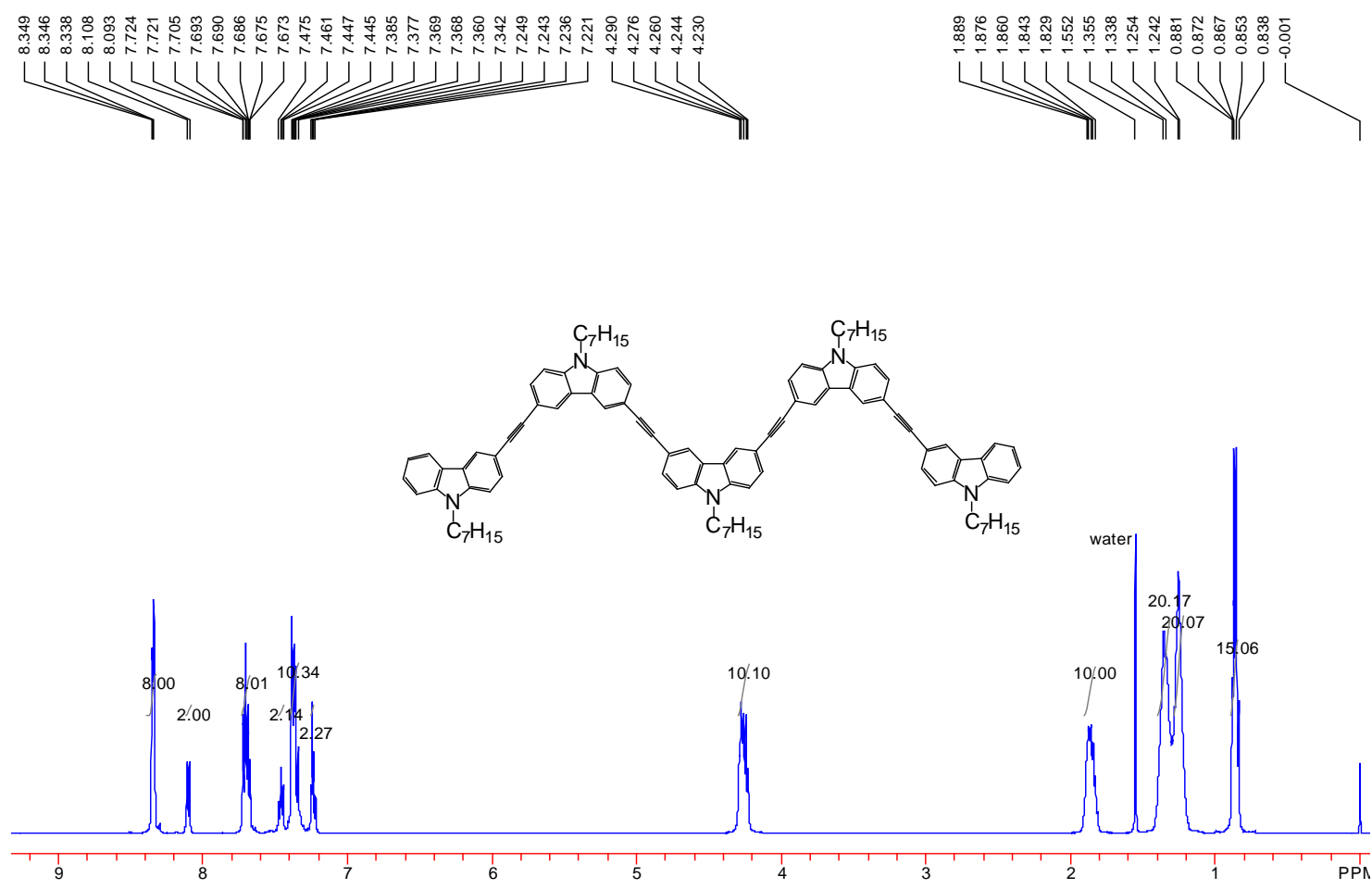

Figure S43: ${ }^{1} \mathrm{H}$ NMR spectrum of compound Cz5.
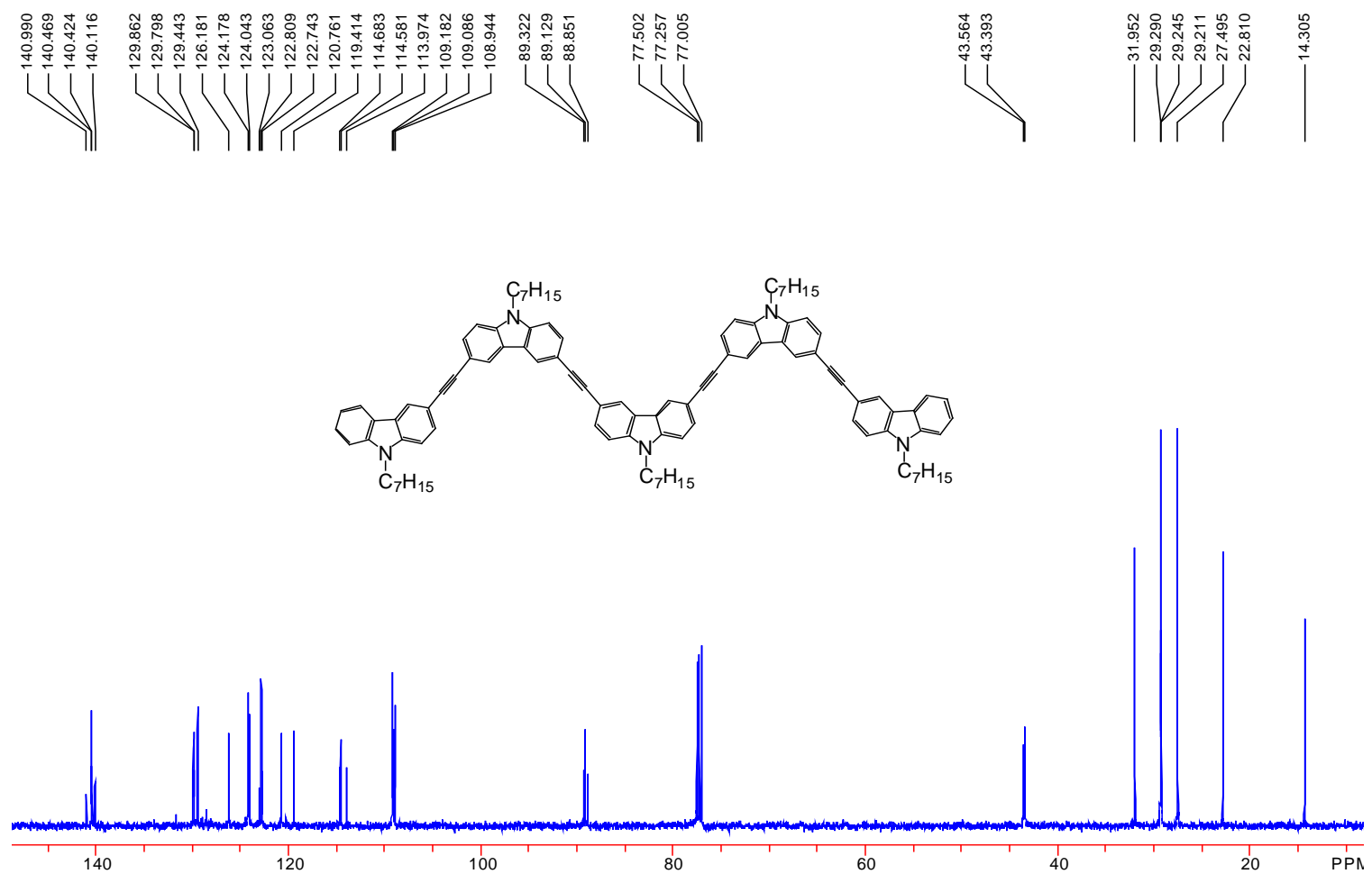

Figure S44: ${ }^{13} \mathrm{C}$ NMR spectrum of compound Cz5. 


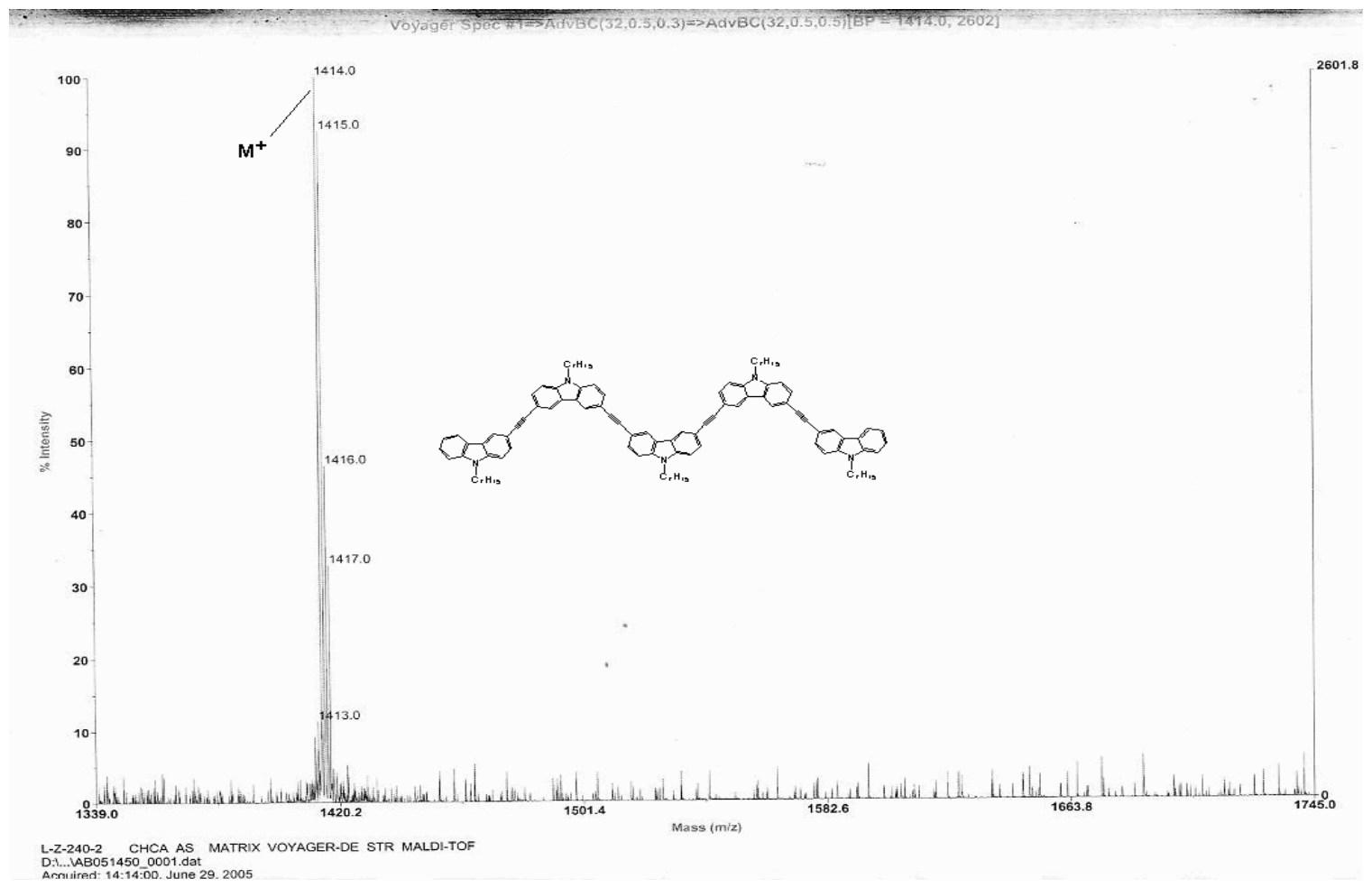

Figure S45: mass spectrum of compound Cz5.
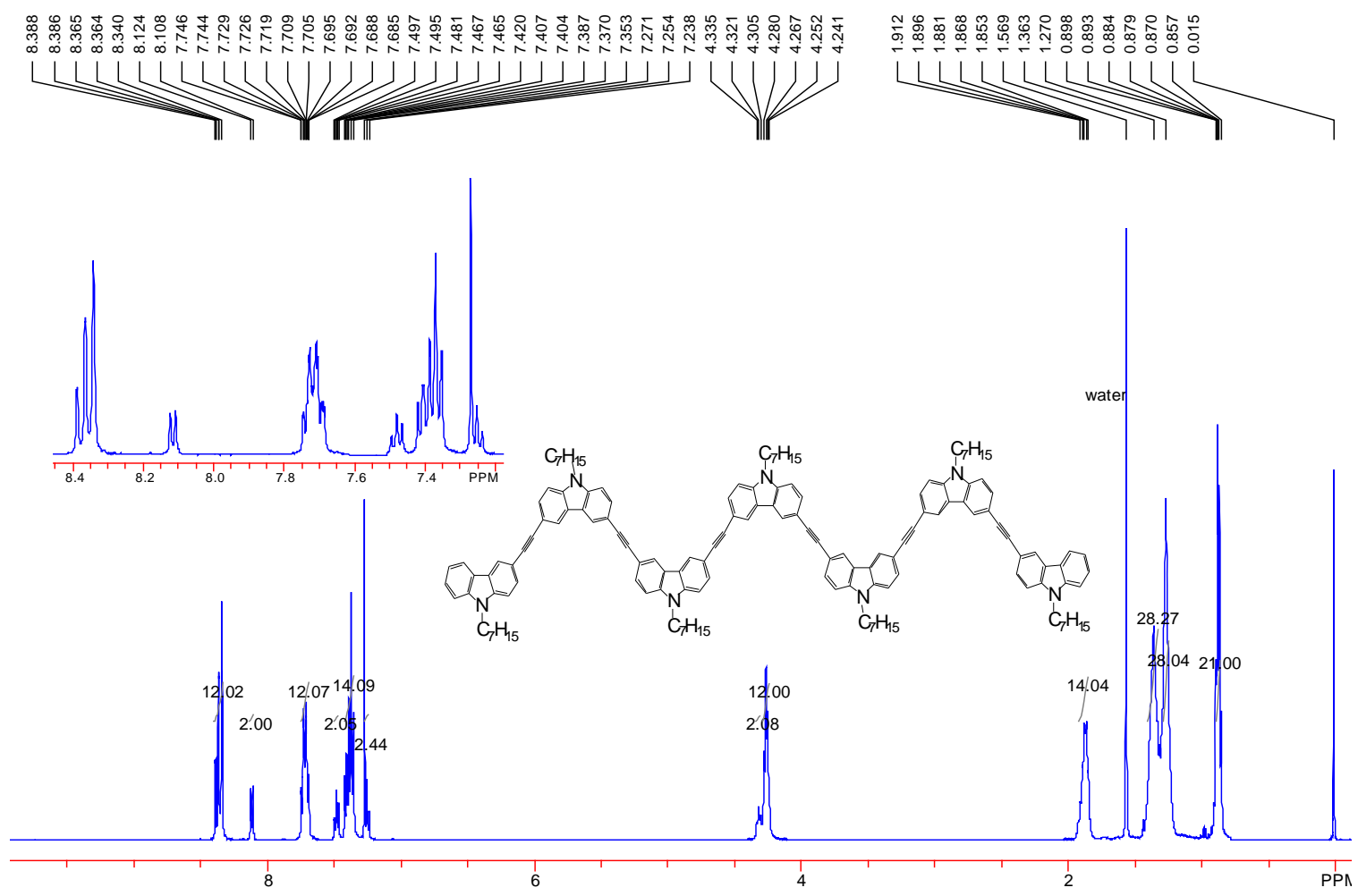

Figure S46: ${ }^{1} \mathrm{H}$ NMR spectrum of compound Cz5. 

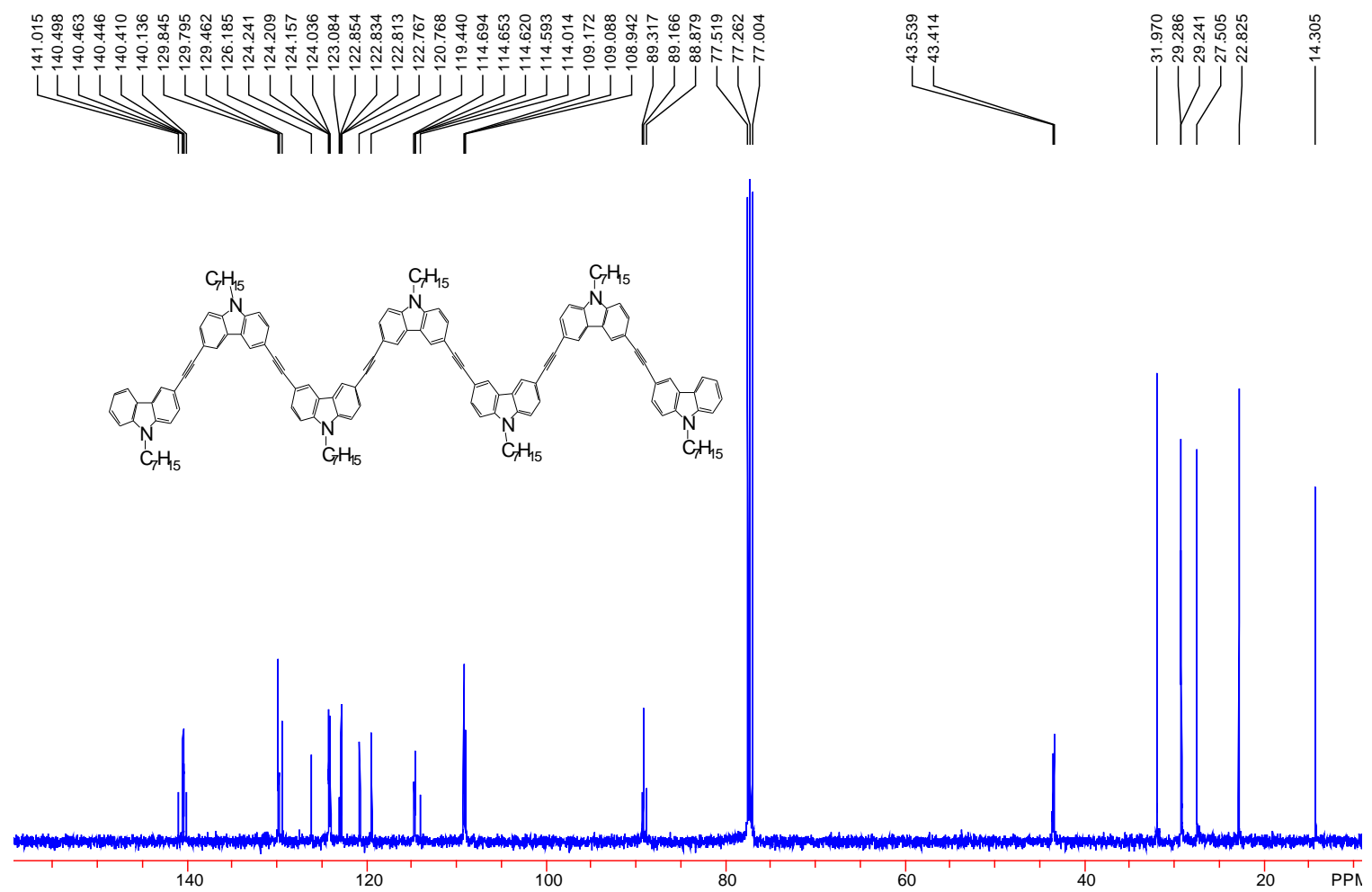

Figure S47: ${ }^{13} \mathrm{C}$ NMR spectrum of compound Cz5.

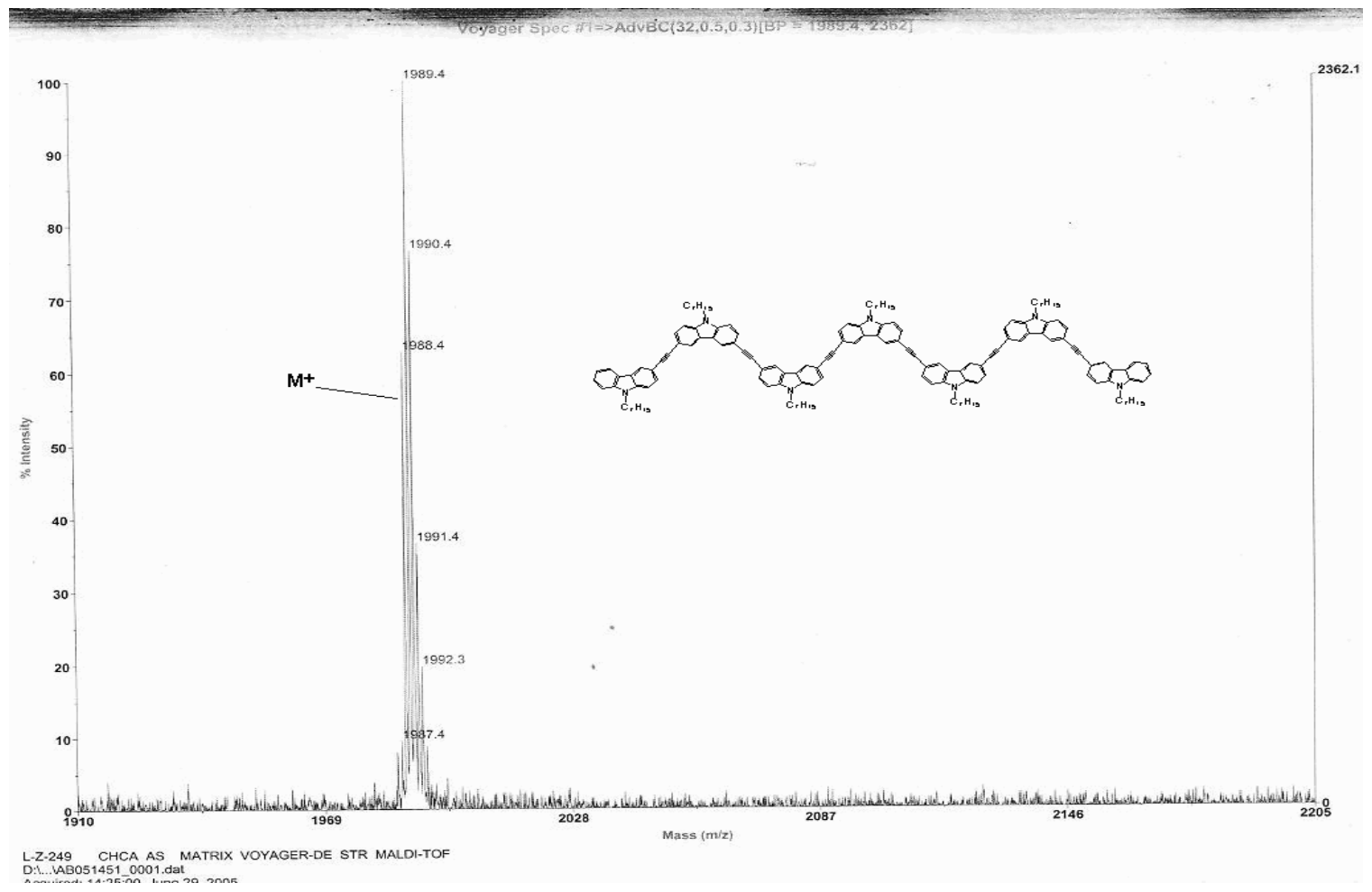

Figure S48: mass spectrum of compound Cz5. 


\section{$J-V-L$ characteristics and efficiency spectra of OLEDs}

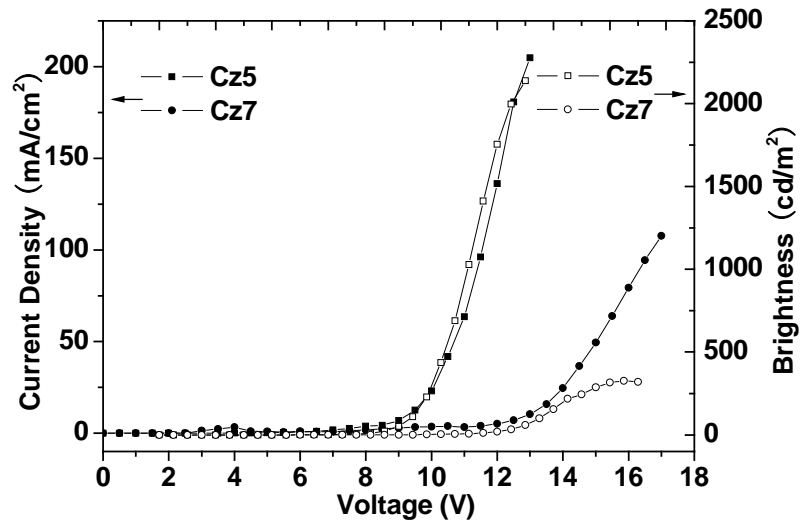

Figure S49. $J-V-L$ characteristics of Type 3 devices based on $\mathbf{C z 5}$ and $\mathbf{C z 7}$.

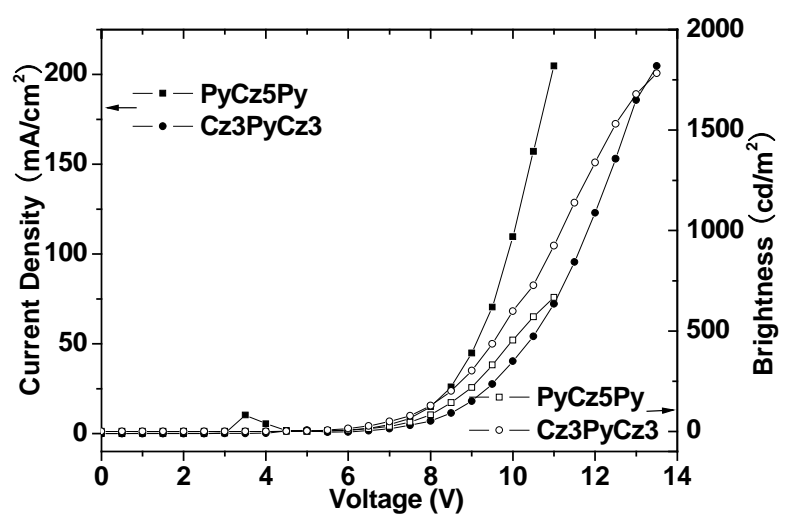

Figure S50. $J-V-L$ characteristics of Type 2 devices based on PyCz5Py and Cz3PyCz3.

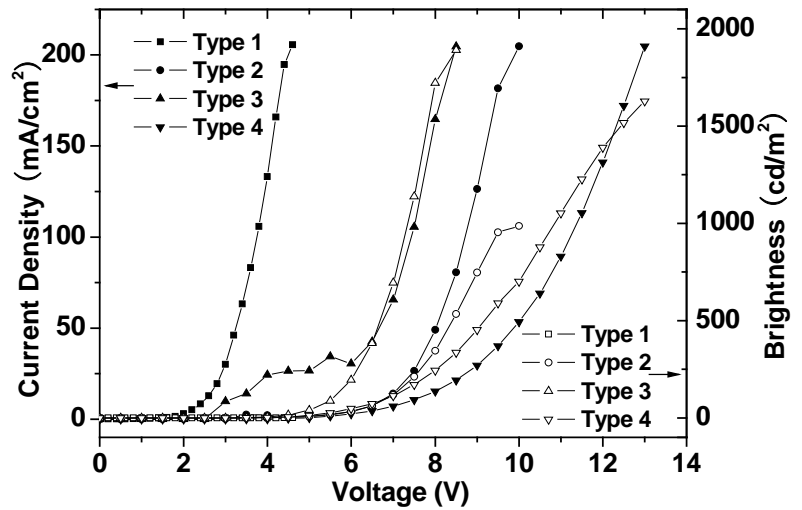

Figure S51. $J-V-L$ characteristics of Type 1-4 devices based on PyCz4. 


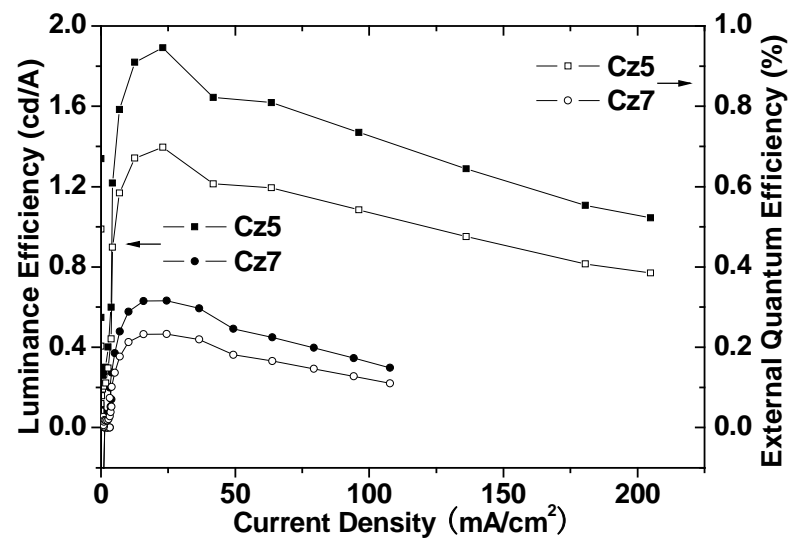

Figure S52: Luminance efficiency and external quantum efficiency of type 3 devices based on $\mathbf{C z 5}$ and Cz7.

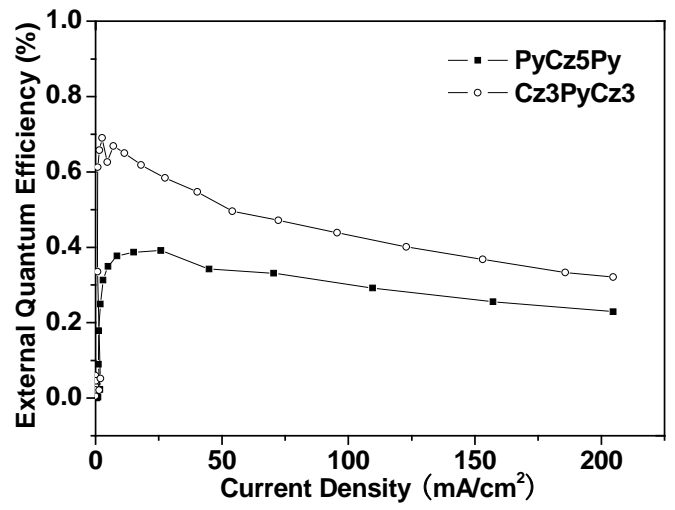

Figure S53: External quantum efficiency of type 2 devices based on PyCz5Py and Cz3PyCz3.

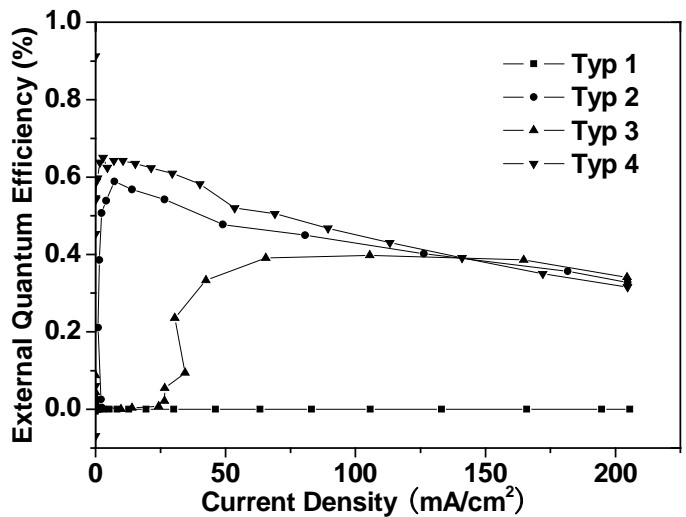

Figure S54: External quantum efficiency of type 1-4 devices based on PyCz4. 